\title{
Consequences of combining life-history traits with sex-specific differences
}

\author{
Vandana Revathi Venkateswaran ${ }^{1, *}$, Olivia Roth ${ }^{2}$ and Chaitanya S. Gokhale ${ }^{1}$ \\ ${ }^{1}$ Research Group for Theoretical Models of Eco-evolutionary Dynamics, \\ Department of Evolutionary Theory, Max Planck Institute for Evolutionary \\ Biology, August Thienemann Str. 2, 24306, Plön, Germany. \\ ${ }^{2}$ Research Group for Parental Investment and Immune Dynamics, \\ GEOMAR-Helmholtz Center for Ocean Research, Düsternbrookerweg 20, \\ D-24105, Kiel, Germany. \\ ${ }^{*}$ Correspondence and request for materials should be addressed to \\ vandana@evolbio.mpg.de
}

\begin{abstract}
Males and females evolved distinct life-history strategies, reflected in diverse lifehistory traits, summarized as sexual dimorphism. Life-history traits are highly interlinked. The sex that allocates more resources towards offspring is expected to increase its life span, and this might require an efficient immune system. However, the other sex might allocate its resources towards ornamentation, and this might have immunosuppressive effects. Activity of immune response may not be specific to the sex that produces the eggs but could correlate with the amount of parental investment given. Informed by experimental data, we designed a theoretical framework that combines multiple lifehistory traits. We disentangled sex-biased life-history strategies from a particular sex to include species with reversed sex-roles, and male parental investment. We computed the lifetime reproductive success from the fitness components arising from diverse sex-biased life-history traits, and observed a strong bias in adult sex ratio depending on sex-specific resource allocation towards life-history traits. Overall, our work provides a generalized method to combine various life-history traits with sex-specific differences to calculate the lifetime reproductive success. This was used to explain certain empirical observations as a consequence of sexual dimorphism in life-history traits.
\end{abstract}

Keywords: Life-history traits, theoretical biology, evolutionary game theory, population dynamics, lifetime reproductive success, adult sex ratio

\section{Introduction}

Fitness is a complicated entity and describes the reproductive success of an individual reflecting the ability of individuals to produce offspring and survive. This arises from trade-offs between various 
life-history traits. Theoretical models assessing the interaction of multiple life-history traits are thus crucial to understand organisms' overall life-history and how they impact fitness. Theoretical and experimental studies have shown how multiple life-history traits define an individual's lifetime reproductive success (Moore, 1990; Martin, 1992; Chapman and Partridge, 1996; Pusey et al., 1997; Fleming et al., 2000; Alonzo, 2002; Kalbe et al., 2009; Alonzo, 2010). However, typically, these traits have been studied in isolation.

In this study, we present a model that addresses the interaction of essential sex-specific life-history traits aiming to obtain the lifetime reproductive success of both sexes. This sheds light on how these traits are contributing to an individual's life-history. We further present the consequences of various sex-specific strategies affecting an evolving population.

Most life-history traits have sex-specific differences. Sex-specific life histories have evolved in the animal kingdom as a consequence of difference in gamete size known as anisogamy (Bell, 1978); females contribute large costly eggs to reproduction and males small cheap sperm. The distinct resource allocation into the offspring asks for sex-specific life-history strategies (Trivers, 1972; Hedrick and Temeles, 1989; Trivers, 2002; Austad, 2006; May, 2007; Roved et al., 2017). Here we focus on the sex-specific differences in three life history traits namely 1 . Parental investment 2. Ornamentation and 3. Immunocompetence

In many species, parental investment is not restricted to sperm and egg production. Parental investment (PI) is any behavioural and physiological investment by a parent provided to the offspring (Trivers, 1972, 2002). The sex that needs to allocate more resources towards the offspring strives for increased longevity since offspring survival also depends on the survival of the parent. Increased longevity requires the allocation of resources into parasite defence and, hence, immunity. Intense costly intrasexual competitions for obtaining mates are performed by allocating resources towards ornamentation (Hillgarth and Wingfield, 1997; Wong and Candolin, 2005; Andersson and Simmons, 2006). To this end, fewer resources may be available for the immune defence in the sex majorly investing in intrasexual interactions. This implies that both ornamentation and parental investment contribute to sexual immune dimorphism (Forbes, 2007; Nunn et al., 2008; Roth et al., 2011; Lin et al., 2016). Thus focusing only on one life-history trait in isolation will not shed light on the individual's true lifetime reproductive success.

We aimed for designing a framework in which multiple life-history traits and their interactions can be studied simultaneously. Particularly, we have constructed a holistic framework that captures sex-specific differences in parental investment, ornamentation and immune response and presents the outcomes of the overall life-history of a sex. We observed two important consequences of sex differences in life history interactions: 1) skewed adult sex ratios and 2) different ratios of homozygous and heterozygous individuals between the sexes with regard to immune alleles. We validated our findings using empirical data from a broad range of animal taxa and diverse life-history strategies to test the limits of our approach.

\section{Model}

We amalgamated approaches from standard population genetics and eco-evolutionary processes (Freeman and Herron, 2007; Otto and Day, 2007; Venkateswaran and Gokhale, 2019) (within and between populations) to investigate the interaction dynamics of multiple life-history traits (with sex-specific differences). We first developed a robust method (illustrated in Figure 1 to study the lifetime reproductive success (LRS) that arises from immune response, mating competition through ornaments and parental investment. Later, we used the LRS to investigate the consequences of combining the sex-specific interactions that are part of an individual's reproductive lifetime. 


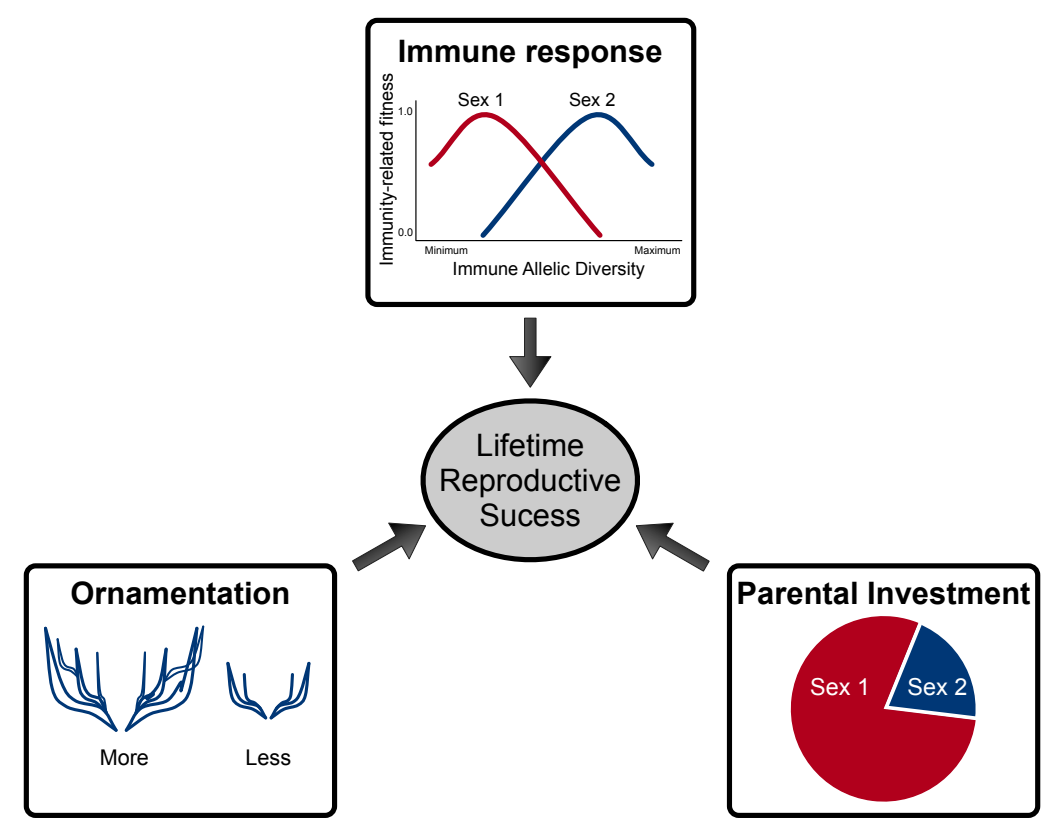

Figure 1: Model representation. Life-history traits affect the lifetime reproductive success. The fitness components from parental investment, immune system and ornamentation are offspring success, survival of the parent plus offspring and mating success, respectively. These contribute to an individual's lifetime reproductive success. We assumed that Sex 1 provides more parental investment $(\mathrm{PI})$ than Sex 2 . The sex-specific fitness from parental investment is modeled as frequency dependent since the number of copulations in one sex depends on the availability of the other sex. The individuals within a sex also have different levels of ornamentation, which they use to attract individuals of the other sex as potential mates. The model uses evolutionary game theory which gives frequency dependent fitnesses of two types of individuals: those with more and those with lesser levels of ornaments. The individuals also differ in their immune genotypes. Each immune genotype yields a certain immunity-related fitness value that depends on the type and number of different immune alleles. The strength of immune response differs between sexes (sexual immune dimorphism). We modeled the evolution of these immune genotypes using population dynamics. Finally, the fitness obtained from parental investment, ornamentation and immune response were used to measure the lifetime reproductive success of an individual.

Consider the two sexes in a population, Sex 1 denoted by a filled circle $\bullet$, and Sex 2 denoted by a diamond $\diamond$. We first consider one autosomal immunity locus $A$ having two alleles $A_{1}$ and $A_{2}$. The three distinct zygotes genotypes would be $A_{1} A_{1}, A_{1} A_{2}$ and $A_{2} A_{2}$. For Sex 1 , which throughout this manuscript does major $\mathrm{PI}$, the frequencies of the three genotypes are denoted by $x_{\bullet 1}, x_{\bullet 2}, x_{\bullet 3}$. The fitnesses, of the same, are denoted by $W_{\bullet 1}, W_{\bullet 2}$ and $W_{\bullet 3}$. Similarly, we denote the frequencies and fitnesses for Sex 2 .

We used standard Mendelian segregation to model the evolution of the different types of individuals in the population. The genotype dynamics following this segregation patterns are denoted in the ESM. As with normal Mendelian segregation we assumed equal sex ratio; half of the offspring are Sex 1 and the other half, Sex 2 . 
A

$\Omega=$ Sex-specific fitness effect of Sex 1 with respect to Sex 2

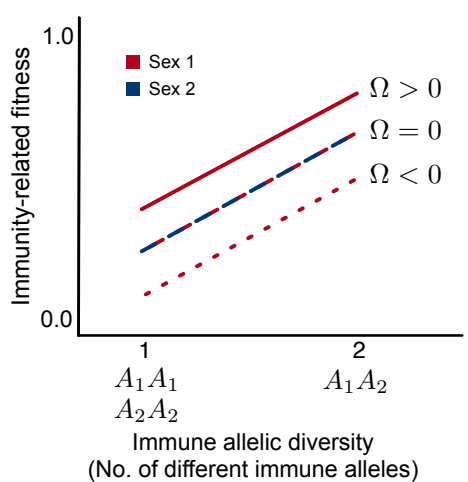

B

$\Theta=$ Effect of diversity on sex-specific fitness of Sex 1 with respect to Sex 2

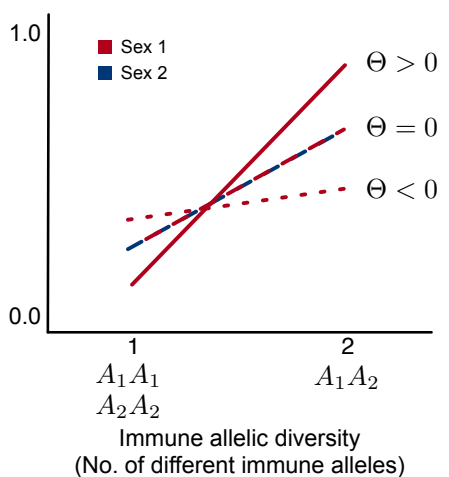

$\Delta=$ Sex-specific fitness effect plus effect of diversity on sex-specific fitness

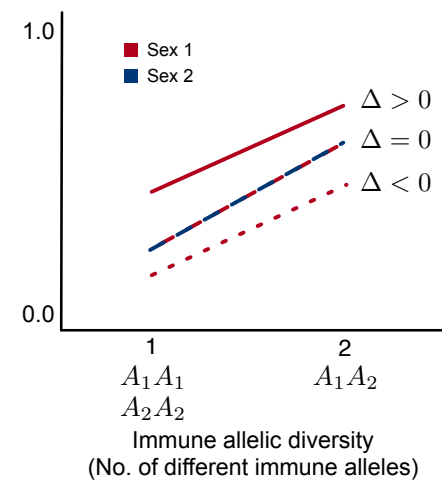

Figure 2: Schematic representation of different scenarios of sex-specific differences in host immunityrelated fitness versus immune allelic diversity. We considered three distinct immune genotypes $A_{1} A_{1}, A_{1} A_{2}$, and $A_{2} A_{2}$ that result from mating between individuals having one immune gene locus $A$ with two alleles $A_{1}$ and $A_{2}$ (Mendelian segregation, see ESM). Fitness positively correlates with the number of different alleles or allelic diversity (Apanius et al., 1997; Eizaguirre et al., 2009). So genotypes $A_{1} A_{1}$ and $A_{2} A_{2}$ (homozygotes) will have the same fitness value as they both have only one type of allele. But $A_{1} A_{2}$ (heterozygote) which has two different types of alleles will have a higher fitness. This is known as heterozygous advantage and occurs within both sexes. However, between the sexes, there can be sex-specific differences (Roved et al., 2017). This is shown in panels (A), (B) and (C). In (A), $\Omega>0$ would imply that Sex 1 will have a higher value of immune response as compared to Sex 2 for any given allelic diversity. When $\Omega<0$, Sex 1 has a lower values of immune response for any given allelic diversity as compared to Sex 2 . Another situation is also possible: Sex 1 can have higher immune response for a homozygous locus, and lower immune response for a heterozygous locus when compared to Sex 2. This shown in (B), where $\Theta$ is the difference between the angles of the two lines. In (C), $\Delta$ differs from $\Omega$ by considering lines that are not parallel to each other i.e. case $C$ is a combination of cases $\mathrm{A}$ and $\mathrm{B}$. When both sexes have the same immune response patterns, $\Omega=\Theta=\Delta=0$.

\section{Fitness}

The lifetime reproductive success i.e. the overall fitness of an individual, is related to its immunocompetence (the ability of an individual to produce a normal immune response following exposure to a pathogen), and offspring success (Stoehr and Kokko, 2006; Kelly and Alonzo, 2010). Thus, in our model the sex-specific fitness components resulting from immune response, ornamentation and parental investment give the lifetime reproductive success of individuals of a sex as shown in Figure 1. Below we introduce the fitness functions independently starting with immunity.

Immune response. A host's immunological diversity helps eliminate a large number of pathogens and disease causing agents. However, in some cases, having too high diversity may reduce efficient immune response e.g. auto-immune diseases triggered by high Major Histocompatibility Complex (MHC) diversity. Thus, having an optimal number of alleles (intermediate diversity) has been shown to be ideal in many systems (Nowak et al., 1992; Milinski, 2006; Woelfing et al., 2009). The host's immunological diversity can be coarsely split up into three parts: low diversity ( $L D$, low efficiency of the immune system), intermediate or optimal diversity ( $I D$, optimal immune efficiency), and high 

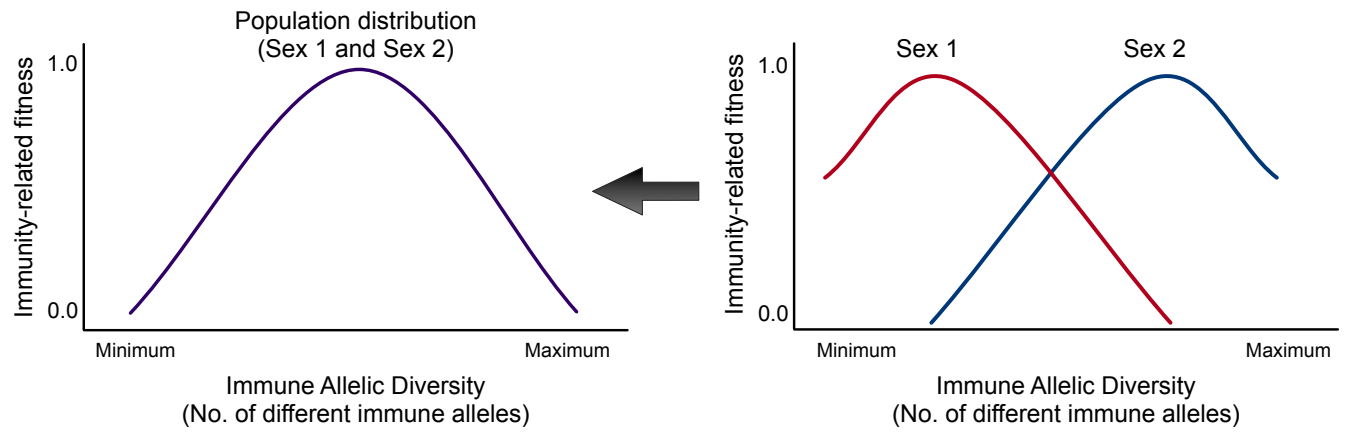

Figure 3: Schematic representation of host immunity-related fitness versus immune allelic diversity. For two immune gene loci $A$ and $B$ each having two alleles $A_{1}, A_{2}$ and $B_{1}, B_{2}$, there would be ten distinct zygote genotypes. The population will comprise of individuals with these genotypes. Their immune responses would depend on these genotypes. The probability of immune response might reduce if the individual has too many immunity allele diversity. In the case of MHC, the auto-immune effect of having high MHC allele diversity reduces the probability of immune response (Nowak et al., 1992; Milinski, 2006; Woelfing et al., 2009). Thus there is an optimal allele diversity, which gives the parabolic shape to the curve. Recent studies have shown that males and females can have different optimal diversities ((Roved et al., 2017, 2018) and Winternitz et al., unpublished). Plotted here are hypothetical sex-specific optima of immune allelic diversity (Roved et al., 2017). The realized population distribution is what is typically looked at, but in our study we consider sex-specific optima of immune allelic diversity. Some immune genes may follow completely different sex-specific patterns from the one shown here (Roved et al., 2017; De Lisle, 2019), and this model can be used for most kinds of immune genes.

diversity ( $H D$, might reduce the efficiency of the immune system). Recent experimental studies by Roved et al. $(2017,2018)$ and Jamie Winternitz and Tobias Lenz (personal communication) show that the optimal diversity could differ between the sexes. Based on these ideas, we have different cases that are shown in the Figure 2 for one immune locus $A$ with two alleles $A_{1}$ and $A_{2}$ that gives three distinct parent and offspring genotypes $A_{1} A_{1}, A_{1} A_{2}$, and $A_{2} A_{2}$ denoted by $j=\{1,2,3\}$. We denote their immune responses by $W_{\bullet j}^{I}$ and $W_{\diamond j}^{I}$ for genotypes $j=\{1,2,3\}$ in the two sexes. In our model, we refer to immune allelic diversity as the number of different immune alleles in the immune loci. A non-linear immune allelic diversity profile shown in Figure 3 where the negative effect of $H D$ is also addressed is considered later.

These approaches can be generalised to any genetic system controlling the immune response or a completely different causal mechanism devoid of the genetic correlation. For example, the effect of nutrients and its effect of the immune system can be captured by a non-genetic model as well (Chandra, 1983). Thus, while we focus on the genetic mechanism in the current model, we stress that our framework is independent of the exact mechanism of how the immune response curves develop. Condition of an individual is directly proportional to immune response (resources allocated to self-maintenance, immune defense) which in turn determines the survivability (Stoehr and Kokko, 2006).

Parental investment. Both sexes pay the costs for initial PI, i.e. egg and sperm production (Hayward and Gillooly, 2011). Pregnancy and parental care vary massively among species (Trivers, 1972; Wade and Shuster, 2002; Trivers, 2002; Kokko and Jennions, 2003; Alonzo, 2010) (Figure 1). We assume that Sex 1 provides major PI (e.g. male sticklebacks, male pipefish, most female 
mammals). The fitness from PI will depend on the relative abundance of the other sex and are given by, $W_{\bullet}^{P}=\left(b^{P}-c_{\bullet}^{P}\right) \cdot \frac{x_{\diamond}}{x_{\bullet}+x_{\diamond}}$ and $W_{\diamond}^{P}=\left(b^{P}-c_{\diamond}^{P}\right) \cdot \frac{x_{\bullet}}{x_{\bullet}+x_{\diamond}}$. Here, $b^{P}$ is the benefit (offspring produced) from $\mathrm{PI}$ while $c_{\bullet}^{P}$ and $c_{\diamond}^{P}$ are the costs for $\mathrm{PI}$ by Sex 1 and Sex 2, respectively. The frequency of Sex 1 equals $x_{\bullet}=x_{\bullet 1}+x_{\bullet 2}+x_{\bullet 3}$ and the frequency of individuals in Sex 2 equals $x_{\diamond}=x_{\diamond 1}+x_{\diamond 2}+x_{\diamond 3}$. Since we have assumed that Sex 1 provides maximum parental investment, $c_{\diamond}^{P}<c_{\bullet}^{P}<b^{P}$.

Ornamentation Mating competitions occur among individuals of the same sex to attract and obtain mates from the other sex. This is performed through fights, nuptial gifts, nests, sexual signals, ornament display and various types of 'attractiveness'. We refer to all of these as 'ornaments'. The investment into the display of ornaments will in most cases rise the chances of acquiring mates (Carranza et al., 1990; Petrie et al., 1991; Berglund et al., 1997; Wong and Candolin, 2005). However, ornamentation is often a costly signal (Zahavi, 1977; Andersson and Simmons, 2006; Milinski, 2006; Kurtz, 2007)). Individual assessment of immune responses helps defining the costs.

When Sex 2 participates in mating competition as shown in Figure 1, two types of Sex 2 individuals were considered in this interaction: one type displays more ornaments $(M O)$ and the other type displays less ornaments $(L O)$. Therefore Sex 2 consists of six types of individuals $-x_{\diamond j, M O}$ and $x_{\diamond j, L O}$ where the genotype $j=\{1,2,3\}$. The frequency-dependent fitness that emerge from these interactions are written as $W_{\diamond M O}^{O}$ and $W_{\diamond M O}^{O}$ (see ESM for details).

\section{Overall dynamics}

The lifetime reproductive success is a multiplicative effect of the fitness arising from immune response, ornamentation and parental investment (Stoehr and Kokko, 2006; Kelly and Alonzo, 2010) as shown in the ESM. Using the LRS values in the Mendelian population dynamics, we can obtain the combined interaction dynamics of each type of individuals in the population (details and calculations in the ESM). The population is divided into nine types of individuals - the three genotypes $(j)$ of Sex $1, x_{\bullet j}$, and the three genotypes of Sex 2 further split according to ornamentation into $x_{\diamond j, M O}$ and $x_{\diamond j, L O}$. We refer to them as simply $x_{i}$ with $i$ as the type of individual. The classical selection equation from population genetics (Crow and Kimura, 1970) gives the evolution of the frequency $x_{i}$ having average fitness $W_{i}$ (Crow and Kimura, 1970; Schuster and Sigmund, 1983; Hofbauer and Sigmund, 1998; Gokhale et al., 2014). The equation can be written as,

$$
\dot{x}_{i}=x_{i}\left(W_{i}-\bar{W}\right)
$$

where $\bar{W}$ is the average population fitness.

\section{Results}

\section{Linear immune allelic diversity profile: single locus}

The diversity levels in the immune alleles can result in differing immune response (e.g. MHC homozygotes and heterozygotes, are known to have different immune responses (Apanius et al., 1997; Eizaguirre et al., 2009)). For one immune locus with two alleles, higher allele diversity boosts the immune response as shown Figure 2. The negative effect of very high diversity is not considered here. Besides the null model of no sexual conflict within the allele diversity, we also include different cases of sexual conflict (Roved et al., 2018) (Figure 2).

When we assume that both the sexes are not involved in mating competition i.e. ornamentation competition game is neutral; we can vary the cost of $\mathrm{PI}$ and the immune response curves (shown in Figure 2). The resulting equilibrium frequencies are shown in Figure 4. When the cost of PI is zero 

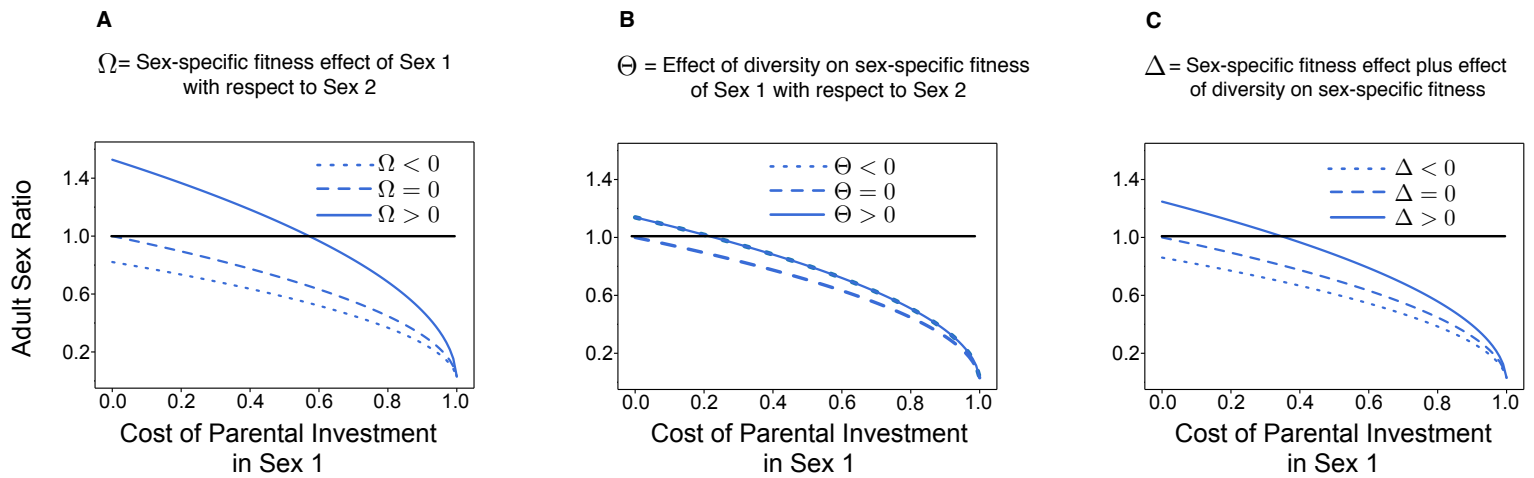

Figure 4: Adult sex ratio (Sex 1: Sex 2) for varying parental investment (PI) and various cases of sexual conflict within immune allelic diversity as shown in Figure 2. The ornamentation game is neutral, i.e. no selection acting on it (details in the ESM). As maintained throughout this study, Sex 1 does maximum PI. Sex 2 does negligible PI. Therefore, its cost is set to zero i.e. $c_{\diamond}^{P}=0$. The black line highlights the even adult sex ratio i.e. 1:1. In (A), (B) and $(\mathrm{C})$ : When the cost of $\mathrm{PI}=0$ and there is no sex difference in immune response $(\Omega=\Delta=\Theta=0)$, the obtained adult sex ratio is $1: 1$. In $(\mathrm{A})$ and $(\mathrm{C})$ : when $\mathrm{Pl}$ increases, the frequency of Sex 1 drops as $\mathrm{PI}$ is costly. When $\Omega>0$ and $\Delta>0$, this sex difference in immune response compensates for the cost of PI. The fall in frequency of Sex 1 is lower than when $\Omega=0$ and $\Delta=0$ and Sex 1 has higher frequency than Sex 2 for most values of PI cost. However, when $\Omega<0$ and $\Delta<0$, Sex 1's frequency decreases with an increase in PI. In (B): Frequency of Sex 1 is lower than Sex 2 for most values of PI cost for most $\Theta$ values. Moreover, $\Theta<0$ and $\Theta>0$ give the same results. The above results highlight the fact that sexual conflict within immune allelic diversity can increase (when $\Omega>0$ and $\Delta>0$ ) or reduce (when $\Omega<0, \delta<0$, almost all $\Theta$ ) the adult sex ratio.

and there is no sex-biased difference in immune response, we observe that the sex ratio is $1: 1$. Here, we focus on the adult sex ratio (ASR) (Kokko and Jennions, 2008). The classical definition of ASR is number of males:total number of males and females, but in our Sex 1 could be male or female. In this manuscript the term ASR is defined as the ratio between Sex 1 and Sex 2. Since in every generation, offspring are produced in equal sex ratios (see ESM), what we obtain is the sex ratio of the offspring after they become adults, perform mating interactions and parental investments. The frequency of Sex 1 decreases with increasing PI. However, Sex 1 increases in frequency under certain cases of sexual conflict over the immune allelic diversity (see $\Delta>0, \Omega>0$, or $\Theta \neq 0$ in Figure 2). The results after including mating competitions are plotted in the figures in the ESM.

Under selection, the obtained genotypes deviated from the Hardy-Weinberg equilibrium (see Figures S.3, S.5 and S.4 of the ESM). One sex has a higher number of heterozygotes when compared to the other sex. In this setup, the heterozygous immune genotype $\left(A_{1} A_{2}\right)$ has a higher immune response than the homozygous genotypes $A_{1} A_{1}$ and $A_{2} A_{2}$ (Figure 3 ). Thus, an increase in heterozygotes within one sex compared to the other would also mean that this sex has a higher mean activity of the immune system. There are scenarios, such as a recent study with wild songbird populations, where the number of heterozygotes and homozygotes even under selection turned out to be equal between the sexes (Roved, 2019). However, this could just be the result of a particular immune response profile, parental investment and ornamentation costs in that species. Different profiles of sexual conflict within the immune allelic diversity would determine different ratios of homozygotes 


\section{Nonlinear diversity profile}

A

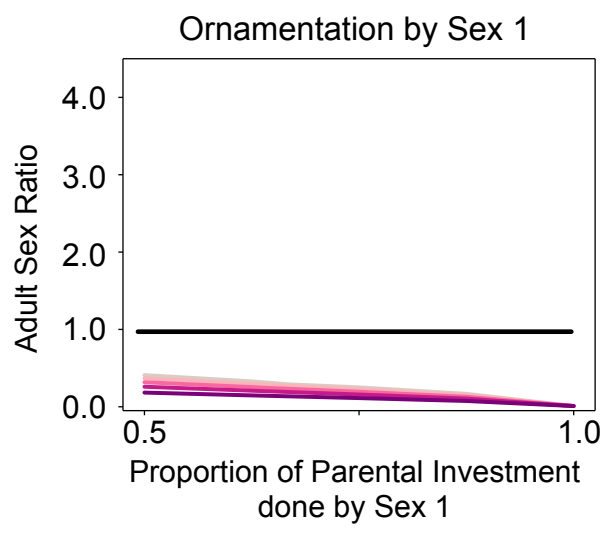

B

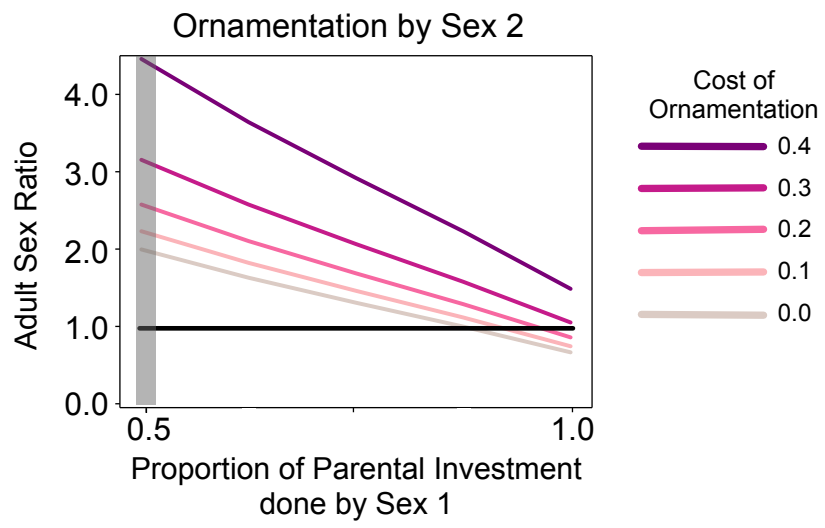

Figure 5: Qualitative difference in the adult sex ratio for diverse polygamous species with varying parental investment $(\mathrm{PI})$ and ornamentation costs. As defined throughout the manuscript, Sex 1 is the major PI provider. For these calculations, we used the sexual conflict Case 4 shown in Figure 3. (A) Species such as sticklebacks where one sex performs both ornamentation and most PI. We observe that frequency of Sex 1 descends as its PI cost increases and this further decreases with a rise in its ornamentation cost. (B) The panel highlighted in gray shows bi-parental investment scenarios. In species where Sex 1 does most $\mathrm{PI}$ and Sex 2 performs elaborate mating competitions, the frequency of Sex 1 reduces with increasing PI. However, this value grows with ascending ornamentation cost in Sex 2. Note that for certain ornamentation and PI values, the adult sex ratios are equal. As shown by previous studies on multiple interactions between traits (Venkateswaran and Gokhale, 2019), even in the case where the cost of ornamentation is equal to zero in the mating competition game, the mere presence of that game will deviate the frequency of Sex 2 from a scenario where there is no ornamentation game.

In a multi-loci scenario, one can include non-linear density profiles (Nowak et al., 1992; Woelfing et al., 2009) as shown in Figure 3. Across species, different sex-specific immune response profiles can be found, depending on the sex-specific selection and phenotypic divergence (Uekert et al., 2006; Love et al., 2008; Oertelt-Prigione, 2012). We hypothesis two such scenarios,

- the optimal diversity of immune alleles for both sexes is the same but the immune responses at this optimal diversity could be different (for instance, females are more prone to acquiring autoimmune diseases; sex hormones such as estrogen, testosterone also affect immune response (Hillgarth and Wingfield, 1997; Törnwall et al., 1999; Whitacre, 2001) or,

- the two sexes have different optimal diversity of immune alleles and the immune response at this optimal diversity is the same for both sexes. For instance, as shown in Roved et al. (2017, 2018), males and females have a different optimal diversity, where males need a higher number of allele diversity to mount maximum immune response. We considered such a scenario for this study (see Figure 3). 
As done for the one locus scenario, we assume that only the number of different alleles i.e. allele diversity produces unequal fitness.

\section{Adult sex ratio in various species}

Our results showed that a sexual conflict within immune allelic diversity and varying parental investment may result in adult sex ratio bias. The effect of ornamentation also plays an important role in skewing adult sex ratios as shown Figure 5. Diverse reproducing species have distinct ornamentation and parental investment costs. Figure 5 shows the values of adult sex ratios that our model predicts for a wide range of species.

\section{Discussion}

Various intersexual and intrasexual interactions during the reproductive lifespan of an individual determine its lifetime reproductive success (Stoehr and Kokko, 2006; Kalbe et al., 2009; Kelly and Alonzo, 2010). We have presented a model framework where several individual life-history interactions can be studied simultaneously. As shown in many empirical studies, the ASR has an impact on sex-specific differences and roles (Liker et al., 2013; Székely et al., 2014; Liker et al., 2015; Henshaw et al., 2019). Our results showed that the interaction of sex-specific life-history traits result in a biased adult sex ratio (ASR) (Pipoly et al., 2015). We showed that the vice versa is also possible (Kokko and Jennions, 2008) i.e., our results showed that ASR is a consequence of sex-specific differences. Our model incorporates the fact that fitness is a complex entity (Doebeli et al., 2017). The overall lifetime reproductive success is a combination of fitness values arising from the individual life-history strategies (here, parental investment, ornamentation and immunocompetence). This model showed that the variation in individuals' or the sex-specific lifetime reproductive success (based on their cost of parental investment, ornamentation and immune response levels) has population level consequences i.e. a skew in adult sex ratio (see Figures S.1 and S.2 in the ESM). Here, the females and males of one generation mate and produce equal numbers of daughters and sons in the next generation. Therefore, at birth, sex ratio of every generation was 1:1. The life-history traits are passed on from parents to offspring. Thus, even though every generation starts with equal sex ratio, their sex-specific traits change the adult sex ratio in every generation until it reaches an equilibrium state.

If a sex does both ornamentation and maximum parental investment, i.e. pays high costs of ornamentation and PI (eg. stickleback males), the ASR will be biased towards the sex that bears negligible costs for ornamentation and PI (e.g. female sticklebacks) (Hagen and Gilbertson, 1973)). Thus, the high costs for contributing to both PI and ornamentation cannot be compensated (Daly, 1978) (Figure 5.A).

In birds and free-spawning fish both sexes exhibit similar levels of parental investment (equally little parental investment by both sexes in case of free-spawning fish) (Perrone Jr and Zaret, 1979; Gross and Sargent, 1985; Cockburn, 2006). Our model shows that these species could show equal ASR for certain parental investment and ornamentation levels (see Figure 5.B). However, in species where males have a higher ornamentation level, the ASR will be biased. For instance, free-spawning species such as the Atlantic salmon where males have elaborate ornaments, show a high adult sex ratio (7:1 ratio of males to females) (Mobley et al., 2019). Therefore, the high sex ratio values shown in the gray shaded region of Figure 5.B matches natural observations.

When one sex does maximum parental investment while the other displays ornaments, ASR is biased towards the sex that does more parental investment, as the other sex has to pay the costs of ornament display (Figure 5.B). Consider the pipefish species $N$. ophidion where males glue the 
eggs on the belly and thus perform partial parental investment (Berglund et al., 1986). In contrast to pipefish species with placenta-like structure and an active transfer of nutrients and oxygen to the embryo (e.g. S. typhle (Berglund et al., 1986; Smith and Wootton, 1999)), N. ophidion only provide partial parental investment. We thus expect a decrease in frequency of $S$. typhle males compared to $N$. ophidion males (Berglund and Rosenqvist, 2003). However, with increasing ornamentation in females the frequency of males increases. Ornaments are costly as they make the bearer more vulnerable to predation. According to Bateman's principle (Bateman, 1948), the reproductive success of the sex that performs mating competition depends on the number of mating events. The sex limited by parental investment will have to live longer for more reproductive events to achieve the same reproductive success as the males (Roth et al., 2011). Thus sex differences in parental investment, ornamentation and immunity (Trivers, 1972; Hedrick and Temeles, 1989; Trivers, 2002; Roved et al., 2017) may also give rise to sexual differences in longevity, an important life-history trait (Austad, 2006; May, 2007).

Our model can be used to determine the lifetime reproductive success using fitness arising from sex-specific differences in life-history traits of a particular sex in a population e.g. parental investment, ornamentation and immunocompetence. Studying the combined dynamics of life-history traits highlights population level consequences such as skewed adult sex ratio (Trivers, 2002; Kokko and Jennions, 2008) emerging due to sex-specific diferences in life-history traits. With the aid of more empirical work directed towards investigating sexual conflict within the immune allelic diversity and other life-history strategies, we can obtain deeper understanding of the overall life-history of a sex or species. Disruptive selection leads to sexual dimorphism and in models that use tools like adaptive dynamics, traits that go through evolutionary branching may end up as two sex-specific traits i.e. sexual dimorphism. Recent studies addressed how coevolution of traits and resource competition drive the evolution of sexual dimorphism (Bolnick and Doebeli, 2003; Stoehr and Kokko, 2006; Vasconcelos and Rueffler, in press). Work by Vasconcelos and Rueffler (in press) demonstrated that even weak trade-offs between life-history traits can result in evolutionary branching that leads to evolution of two co-existing types. In this study, we investigated the eco-evolutionary consequences of interplay between two or more sex-specific life-history traits. Along with empirical evidence that matches our qualitative predictions, suggesting a skewed adult sex-ratio.

The functions in our model that describe fitness from parental investment and ornamentation consider polygamous species. While many sexually reproducing animals are polygamous, species like seahorses are monogamous throughout their lifetime (Vincent and Sadler, 1995). The tradeoffs between ornamentation, parental investment and immunocompetence in monogamous species would be different. For instance, they may not have to bear costs of attracting mates after one brooding season. Our model can be modified to study the effect of integrating monogamous mating patterns. Also, with regard to immune genes such as the ones of the MHC, genetically dissimilar individuals mate more often as the evolutionary incentive is to produce optimal MHC diversity offspring (Milinski, 2006; Woelfing et al., 2009; Kalbe et al., 2009; Eizaguirre et al., 2009). To this end, mating is not random. Aspects of a model by Kirkpatrick (Kirkpatrick, 1982) for two autosomal loci with female mating preference for a trait that occurs in males is a potential extension of our model. Finally, novel studies directed at sexual conflict within the MHC and other immune genes as done by Roved et al. (2018) shall be very beneficial in providing further knowledge of how sex-specific immune defences manifest in different systems with distinct sex-specific ornamentation and parental investment patterns. 


\section{Electronic Supplementary material}

\section{S.1 One locus, two alleles}

Let the fitnesses of the three genotypes be $A_{1} A_{1}, A_{1} A_{2}$ and $A_{2} A_{2}$ are $W_{1}, W_{2}$ and $W_{3}$. The frequencies of the three genotypes are denoted by $x_{1}, x_{2}$ and $x_{3}$ Thus,

$$
p(t+1)=x_{1}+\frac{1}{2} x_{2}=p(t)^{2} \cdot \frac{W_{1}}{\bar{W}}+\frac{1}{2} \frac{2 p(t) q(t) \cdot W_{2}}{\bar{W}} .
$$

Similarly,

$$
q(t+1)=x_{3}+\frac{1}{2} x_{2}=q(t)^{2} \cdot \frac{W_{3}}{\bar{W}}+\frac{1}{2} \frac{2 p(t) q(t) \cdot W_{2}}{\bar{W}} .
$$

The $W$ s could be survivability (viability) or fertility or both. Under neutrality they are all equal to unity (Otto and Day, 2007).

\section{S.2 Separate population into males and females}

If the population is separated into the two sexes, Sex 1 which could be male (or female) denoted by a solid circle symbol $\bullet$, and Sex 2 which could be female (or male) denoted by a diamond symbol $\diamond$. We stick to calling the sexes as Sex 1 and Sex 2 instead of males and females (and we also do not use the standard $q$ and $\sigma^{\prime \prime}$ symbols as it might be misleading) because we want to show a generalized idea of the dependence of sexual immune dimorphism on the amount of parental investment (or mating competition and other factors) given and not to the sex itself.

For Sex 1, let frequency of $A_{1} A_{1}=x_{\bullet 1}$, frequency of $A_{1} A_{2}=x_{\bullet 2}$ and frequency of $A_{2} A_{2}=x_{\bullet}$. Similarly, for Sex 2 , let frequency of $A_{1} A_{1}=x_{\diamond 1}$, frequency of $A_{1} A_{2}=x_{\diamond 2}$ and frequency of $A_{2} A_{2}=x_{\diamond 3}$.

In Sex 1 , let the fitness of individuals with genotype $A_{1} A_{1}=W_{\bullet 1}$, fitness of $A_{1} A_{2}=W_{\bullet 2}$ and fitness of $A_{2} A_{2}=W_{\bullet 3}$. Similarly, for Sex 2 , let the fitness of individuals with genotype $A_{1} A_{1}=W_{\diamond 1}$, fitness of $A_{1} A_{2}=W_{\diamond 2}$ and fitness of $A_{2} A_{2}=W_{\diamond 3}$. The sex that performs mating competitions (say, Sex 2) is further divided into individuals with Less or More Ornamentation ( $L O$ or $M O$ ). Through Mendelian population dynamics we obtain the of frequency of each genotype at subsequent generations (Gokhale et al., 2014),

$$
\begin{aligned}
\bar{W} x_{\bullet 1}^{\prime} & =\frac{W \bullet 1}{2}\left(x_{\bullet 1} x_{\diamond 1}+\frac{x_{\bullet 1} x_{\diamond 2}}{2}+\frac{x_{\bullet 2} x_{\diamond 1}}{2}+\frac{x_{\bullet 2} x_{\diamond 2}}{4}\right) \\
\bar{W} x_{\bullet 2}^{\prime} & =\frac{W_{\bullet 2}}{2}\left(x_{\bullet 1} x_{\diamond 3}+x_{\bullet 3} x_{\diamond 1}+\frac{x_{\bullet 1} x_{\diamond 2}}{2}\right. \\
& \left.+\frac{x_{\bullet 2} x_{\diamond 1}}{2}+\frac{x_{\bullet 3} x_{\diamond 2}}{2}+\frac{x_{\bullet 2} x_{\diamond 2}}{2}+\frac{x_{\bullet 2} x_{\diamond 3}}{2}\right) \\
\bar{W} x_{\bullet 3}^{\prime} & =\frac{W_{\bullet 3}}{2}\left(x_{\bullet 3} x_{\diamond 3}+\frac{x_{\bullet 3} x_{\diamond 2}}{2}+\frac{x_{\bullet 2} x_{\diamond 3}}{2}+\frac{x_{\bullet 2} x_{\diamond 2}}{4}\right) .
\end{aligned}
$$


and

$$
\begin{aligned}
\bar{W} x_{\diamond 1, M O}^{\prime} & =\frac{W_{\diamond 1, M O}}{2}\left(x_{\diamond 1, M O} x_{\bullet 1}+\frac{x_{\diamond 1, M O} x_{\bullet 2}}{2}+\frac{x_{\diamond 2, M O} x_{\bullet 1}}{2}+\frac{x_{\diamond 2, M O} x_{\bullet}}{4}\right) \\
\bar{W} x_{\diamond 2, M O}^{\prime} & =\frac{W_{\diamond 2, M O}}{2}\left(x_{\diamond 1, M O} x_{\bullet 3}+x_{\diamond 3, M O} x_{\bullet 1}+\frac{x_{\diamond 1, M O} x_{\bullet 2}}{2}\right. \\
& \left.+\frac{x_{\diamond 2, M O} x_{\bullet 1}}{2}+\frac{x_{\diamond 3, M O} x_{\bullet 2}}{2}+\frac{x_{\diamond 2, M O} x_{\bullet 2}}{2}+\frac{x_{\diamond 2, M O} x_{\bullet 3}}{2}\right) \\
\bar{W} x_{\diamond 3, M O}^{\prime} & =\frac{W_{\diamond 3, M O}}{2}\left(x_{\diamond 3, M O} x_{\bullet 3}+\frac{x_{\diamond 3, M O} x_{\bullet 2}}{2}+\frac{x_{\diamond 2, M O} x_{\bullet 3}}{2}+\frac{x_{\diamond 2, M O} x_{\bullet 2}}{4}\right) \\
\bar{W} x_{\diamond 1, L O}^{\prime}= & \frac{W_{\diamond 1, L O}}{2}\left(x_{\diamond 1, L O} x_{\bullet 1}+\frac{x_{\diamond 1, L O} x_{\bullet 2}}{2}+\frac{x_{\diamond 2, L O} x_{\bullet 1}}{2}+\frac{x_{\diamond 2, L O} x_{\bullet 2}}{4}\right) \\
\bar{W} x_{\diamond 2, L O}^{\prime}= & \frac{W_{\diamond 2, L O}}{2}\left(x_{\diamond 1, L O} x_{\bullet 3}+x_{\diamond 3, L O} x_{\bullet 1}+\frac{x_{\diamond 1, L O} x_{\bullet 2}}{2}\right. \\
\bar{W} x_{\diamond 3, L O}^{\prime} & =\frac{W_{\diamond 3, L O}}{2}\left(x_{\diamond 3, L O} x_{\bullet 3}+\frac{x_{\diamond 3, L O} x_{\bullet 2}}{2}+\frac{x_{\diamond 2, L O} x_{\bullet 3}}{2}+\frac{x_{\diamond 2, L O} x_{\bullet 2}}{4}\right)
\end{aligned}
$$

where $\bar{W}$ is the average fitness of all genotypes. Also, $x_{\bullet i}^{\prime}$ and $x_{\diamond i}^{\prime}$ is the change in frequencies of the genotypes $i$ (for the different sexes) with time. Also, here we assume equal sex ratio; half of the offspring are males and the other half, females.

Now, let $W_{\diamond 1}=W_{\bullet 1}=W_{1}, W_{\diamond 2}=W_{\bullet 2}=W_{2}$ and $W_{\diamond 3}=W_{\bullet 3}=W_{3}$. where $W_{\diamond i}=$ $W_{\diamond i, M O}+W_{\diamond i, L O}$. Then,

$$
\begin{aligned}
\bar{W} x_{1}^{\prime}=\bar{W} x_{\diamond 1}^{\prime}+\bar{W} x_{\bullet 1}^{\prime} & =W_{1}\left[\left(x_{\bullet 1} \cdot x_{\diamond 1}\right)+\frac{\left(x_{\bullet 1} \cdot x_{\diamond 2}\right)}{2}+\frac{\left(x_{\bullet 2} \cdot x_{\diamond 1}\right)}{2}+\frac{\left(x_{\bullet 2} \cdot x_{\diamond 2}\right)}{4}\right] \\
\bar{W} x_{2}^{\prime}=\bar{W} x_{\diamond 2}^{\prime}+\bar{W} x_{\bullet 2}^{\prime} & =W_{2}\left[\left(x_{\bullet 1} \cdot x_{\diamond 3}\right)+\left(x_{\bullet 3} \cdot x_{\diamond 1}\right)+\frac{\left(x_{\bullet 1} \cdot x_{\diamond 2}\right)}{2} \frac{\left(x_{\bullet 2} \cdot x_{\diamond 1}\right)}{2}\right. \\
& \left.+\frac{\left(x_{\bullet 3} \cdot x_{\diamond 2}\right)}{2}+\frac{\left(x_{\bullet 2} \cdot x_{\diamond 2}\right)}{2}+\frac{\left(x_{\bullet 2} \cdot x_{\diamond 3}\right)}{2}\right] \\
\bar{W} x_{3}^{\prime}=\bar{W} x_{\diamond 3}^{\prime}+\bar{W} x_{\bullet 3}^{\prime}= & W_{3}\left[\left(x_{\bullet 3} \cdot x_{\diamond 3}\right)+\frac{\left(x_{\bullet 3} \cdot x_{\diamond 2}\right)}{2}+\frac{\left(x_{\bullet 2} \cdot x_{\diamond 3}\right)}{2}+\frac{\left(x_{\bullet 2} \cdot x_{\diamond 2}\right)}{4}\right] .
\end{aligned}
$$

\section{S.3 Mating competition}

Mating competition is performed through fights, sexual signals, nuptial gifts, ornament display and various types of attractiveness. We shall refer to all of these as 'ornaments'. Let's assume there are individuals of two types in this interaction: ones that display more ornaments (MO) and ones that display less (LO). Consider the mating competition interaction between individuals of Sex 2 . For the three different genotypes $i$ the population in Sex 2 will consist of six different kinds of individuals, $x_{\diamond j, M O}$ and $x_{\diamond j, L O}$.

We model this interaction as an evolutionary game (Maynard Smith, 1986; Sigmund and Nowak, 1999). The payoff matrix is written as,

$$
\begin{array}{lcc}
M O & L O \\
\text { More Ornament or } M O \\
\text { Less Ornament or } L O
\end{array}\left(\begin{array}{cc}
\frac{b^{O}}{2}-c_{\diamond}^{O} & b^{O}-c_{\diamond}^{O} \\
0 & \frac{b^{O}}{2}
\end{array}\right)
$$


where $b^{O}$ is the benefit arising from mating competitions i.e. mating gain and $c_{\diamond}^{O}$ is the cost that Sex 2 bears to maintain ornament(s). The frequency dependent fitnesses resulting from these interactions are given by,

$$
\begin{aligned}
W_{\diamond M O}^{O} & =\left(\frac{b^{O}}{2}-c_{\diamond}^{O}\right) \frac{x_{\diamond, M O}}{x_{\diamond, M O}+x_{\diamond, L O}}+\left(b^{O}-c_{\diamond}^{O}\right) \frac{x_{\diamond, L O}}{x_{\diamond, M O}+x_{\diamond, L O}} \\
W_{\diamond L O}^{O} & =0 \frac{x_{\diamond, M O}}{x_{\diamond, M O}+x_{\diamond, L O}}+\frac{b^{O}}{2} \frac{x_{\diamond, L O}}{x_{\diamond, M O}+x_{\diamond, L O}}
\end{aligned}
$$

where $x_{\diamond, M O}=\sum_{j=1}^{3} x_{\diamond j, M O}$ and $x_{\diamond, L O}=\sum_{j=1}^{3} x_{\diamond j, L O}$ and $c^{O}<\frac{b_{\diamond}^{O}}{2}$.

The payoff matrix (S.6) is an interaction between a pair of individuals i.e. two player game. We can extend this to $d$-players (Gokhale and Traulsen, 2014; Chen et al., 2017) and the payoffs are given by,

$$
\begin{aligned}
& P_{M O}= \begin{cases}b^{O}-c_{\diamond}^{O} & k=1 \\
\frac{b^{O}}{k}-c_{\diamond}^{O} & k>1\end{cases} \\
& P_{L O}= \begin{cases}\frac{b^{P}}{n} & k=0 \\
0 & k>0\end{cases}
\end{aligned}
$$

where $k$ is the number of $M O$ (More Ornament) players and $n$ is the total number of players. $k$ and $n$ can vary between the sexes.

\section{S.4 Dynamics}

\section{S.4.1 One locus}

If we consider that Sex 1, the sex undergoes major parental investment does not involve in mating competitions and individuals of Sex 2 perform mating competitions, then the population will have nine types of individuals $-x_{\bullet i}, x_{\diamond i, M O}$ and $x_{\diamond i, L O}$ for the three genotypes $i$. We shall refer to them as $x_{1}, x_{2}, x_{3}, x_{4}, x_{5}, x_{6}, x_{7}, x_{8}$ and $x_{9}$.

The lifetime reproductive success of each type within a sex is a multiplicative combination of mating gains, fertility and survival probability (Stoehr and Kokko, 2006; Kelly and Alonzo, 2010). These are given by,

$$
\begin{gathered}
W_{\bullet 1}=\frac{W_{\bullet}^{P}}{1-W_{\bullet 1}^{I}} \quad W_{\bullet 2}=\frac{W_{\bullet}^{P}}{1-W_{\bullet 2}^{I}} W_{\bullet 3}=\frac{W_{\bullet}^{P}}{1-W_{\bullet 3}^{I}} \\
W_{\diamond 1, M O}=\frac{W_{\diamond}^{P} \cdot W_{\diamond M O}^{O}}{1-W_{\bullet 1}^{I}} W_{\diamond 2, M O}=\frac{W_{\diamond}^{P} \cdot W_{\diamond M O}^{O}}{1-W_{\bullet 2}^{I}} \quad W_{\diamond 3, M O}=\frac{W_{\diamond}^{P} \cdot W_{\diamond M O}^{O}}{1-W_{\bullet 3}^{I}} \\
W_{\diamond 1, L O}=\frac{W_{\diamond}^{P} \cdot W_{\diamond L O}^{O}}{1-W_{\bullet 1}^{I}} W_{\diamond 2, L O}=\frac{W_{\diamond}^{P} \cdot W_{\diamond L O}^{O}}{1-W_{\bullet 2}^{I}} \quad W_{\diamond 3, L O}=\frac{W_{\diamond}^{P} \cdot W_{\diamond L O}^{O}}{1-W_{\bullet 3}^{I}} .
\end{gathered}
$$

Here, $W_{\bullet i}^{I}$ and $W_{\diamond i}^{I}$ are the fitness from immune responses (survivability) of type $i$ for Sex 1 and Sex 2 as described in the main text. Similarly, $W_{\bullet}^{P}$ and $W_{\diamond}^{P}$ are the fitness that arise from parental investments performed by members of Sex 1 and Sex 2, respectively. The fitness from More and Less ornamentation ( $W_{M O}^{O}$ and $W_{L O}^{O}$ are as defined in the previous section. Using equations (S.3) and (S.4), we can obtain the average fitnesses for each type of individuals in the population. For Sex 1 they are given by, 


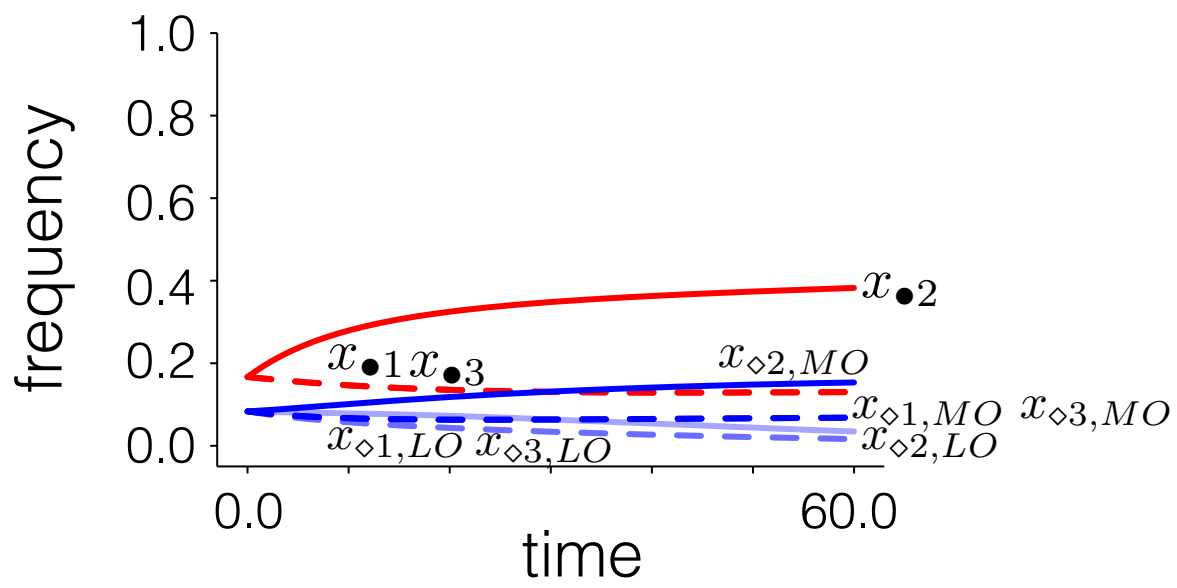

Figure S.1: Evolution of frequency of all possible type of individuals in a population that exhibits sexual dimorphism in immunity and ornamentation, and sex difference in parental investment. When sex 1 performs major parental investment and individuals of Sex 2 perform mating competitions, then the population will have nine types of individuals $-x_{\bullet i}, x_{\diamond i, M O}$ and $x_{\diamond i, L O}$ for the three immunity genotypes $i$. For the results shown in this figure, $c_{\bullet}^{P}=0.6, c_{\diamond}^{P}=0$ and $c_{\diamond}^{O}=0.4$. Fitness from immune response comes from $\Omega>0$ of the linear immune allelic diversity vs immune response profile in Figure 2 in the main article. Red lines are for Sex 1 and blue for Sex 2 . The solid lines are for the heterozygous genotype and dashed lines for the homozygotes. The lighter blue lines in Sex 2 are for individuals with low ornamentation.

$$
W_{\bullet j}=\frac{\bar{W} x_{\bullet j}^{\prime}}{x_{\bullet j}}
$$

where $j=\{1,2,3\}$ and $\bar{W}$ is the average fitness of all types. For Sex 2 they are given by,

$$
\begin{aligned}
W_{\diamond j, M O} & =\frac{\bar{W} x_{\diamond j, M O}^{\prime}}{x_{\diamond j, M O}} \\
W_{\diamond j, L O} & =\frac{\bar{W} x_{\diamond j, L O}^{\prime}}{x_{\diamond j, L O}}
\end{aligned}
$$

where again $j=\{1,2,3\}$. Here, $M O$ and $L O$ correspond to individuals with more and less ornamentation, respectively. From equations (S.11) and (S.11) we know that there are nine different types of individuals whose frequencies can be just described by $x_{i}$ for $i=\{1,2,3, \ldots 9\}$ and their respective average fitnesses are denoted by $W_{i}$ (for $i=\{1,2,3, \ldots 9\}$ ).

Using the above given equations we have,

$$
x_{i}^{\prime}-x_{i}=\left(\frac{x_{i} W_{i}}{\bar{W}}\right)-x_{i} .
$$

The classical selection equation (Crow and Kimura, 1970; Hofbauer and Sigmund, 1998) that gives the evolution of each type (see Figure S.1) is then obtained by taking the time derivative of (S.13) given by, 


$$
\dot{x}_{i}=x_{i}\left(W_{i}-\bar{W}\right) .
$$

The frequencies of all types reach an equilibrium value at some time point. This is our value of interest that is used in the results throughout this ESM and the main article.

The frequencies of each sex is a summation of frequencies of all types of individuals in a sex. Figure S.2 shows how the frequency of the sexes changes with sex-specific differences in immunocompetence, parental investment, and ornamentation.

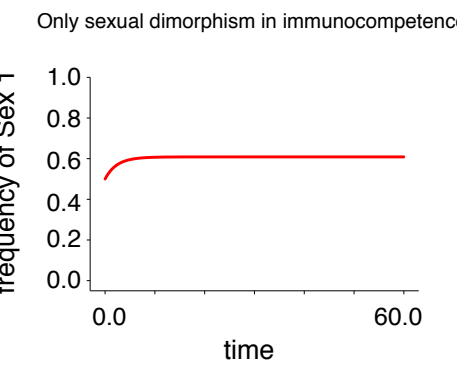

B

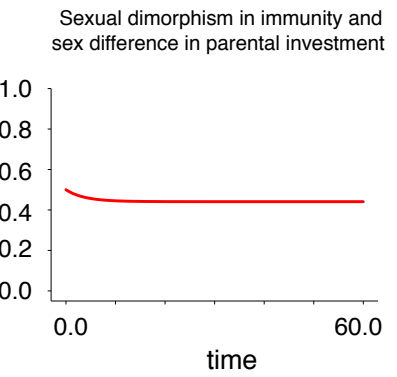

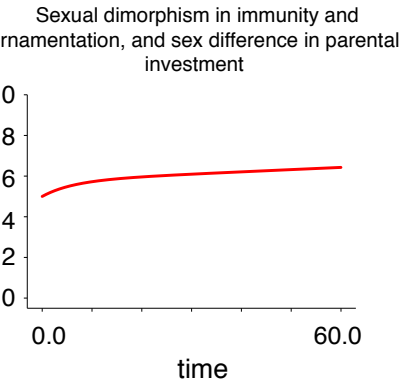

Figure S.2: Evolution of frequency of Sex 1 in the population. Since frequency of $\operatorname{Sex} 1\left(x_{\bullet}\right)$ and frequency of Sex $2\left(x_{\diamond}\right)$ equals unity, $x_{\diamond}$ equals $1-x_{\bullet}$. These frequencies are obtained by summing up all types of individuals within the sexes. (A) When Sex 1 has a higher values of immune response a compared to Sex 2 for all immune allelic diversity $(\Omega)$. (B) When condition $A$ is met, but Sex 1 also performs parental investment, while Sex 2 does not. (C) When conditions $A$ and $B$ are met, and Sex 2 also exhibits ornamentation. The sex-specific traits evolve over generation (time) by selection and therefore, get passed on to subsequent generations (for example, case $C$ is shown in Figure S.1). Therefore, even when the sex ratio is kept equal among offspring at every generation, their sex-specific characteristics change their frequency in the population.

\section{S.4.2 One locus: Results}

\section{Heterozygosity vs Homozygosity}

Under Hardy Weinberg or when all interactions are neutral, the number of heterozygous and homozygous individuals within a sex would be equal. However, under selection (through different probabilities of immune response for homozygotes and heterozygotes), varying cost of parental investment and ornamentation the number of heterozygotes and homozygotes would deviate from neutrality. An increase in heterozygotes within one sex compared to the other, would also mean than it has a higher immune response on average. When we allow for selection to act on all the three factors (parental investment, immunity genes and ornamentation), we can observe their combined effect on the increase in frequency of heterozygous individuals within a sex (results shown in Figures S.3,S.4 and S.5). 


\section{S.5 Two loci having two alleles each}

\section{S.5.1 Population dynamics with separation of population into males and females}

For Sex 1 , let the frequency of $A_{1} B_{1} \mid A_{1} B_{1}=f_{\bullet}\left(A_{1} B_{1} \mid A_{1} B_{1}\right)=x_{\bullet 1}, f_{\bullet}\left(A_{1} B_{1} \mid A_{1} B_{2}\right)=x_{\bullet 2}$, $f_{\bullet}\left(A_{1} B_{2} \mid A_{1} B_{2}\right)=x_{\bullet 3}, f_{\bullet}\left(A_{1} B_{1} \mid A_{2} B_{1}\right)=x_{\bullet 4}, f_{\bullet}\left(A_{1} B_{2} \mid A_{2} B_{1}\right)=x_{\bullet 5}, f_{\bullet}\left(A_{1} B_{2} \mid A_{2} B_{2}\right)=x_{\bullet 6}$, $f_{\bullet}\left(A_{2} B_{1} \mid A_{2} B_{1}\right)=x_{\bullet 7}, f_{\bullet}\left(A_{2} B_{1} \mid A_{2} B_{2}\right)=x_{\bullet}, f_{\bullet}\left(A_{2} B_{2} \mid A_{2} B_{2}\right)=x_{\bullet}$ and $f_{\bullet}\left(A_{1} B_{1} \mid A_{2} B_{2}\right)=$ $x_{\bullet 10}$. Similarly, for Sex 2 .

From Mendelian population dynamics (as done in the one locus case), the frequency of the homozygotes in Sex 1 will be:

$$
\begin{aligned}
\bar{W} x_{\bullet 1}^{\prime} & =\frac{W_{1}}{2}\left(x_{\bullet 1} \cdot x_{\diamond 1}+\frac{x_{\bullet 1} \cdot x_{\diamond 2}+x_{\bullet 1} \cdot x_{\diamond 4}+x_{\bullet 1} \cdot x_{\diamond 10}+x_{\bullet 2} \cdot x_{\diamond 1}+x_{\bullet 4} \cdot x_{\diamond 1}+x_{\bullet 10} \cdot x_{\diamond 1}}{2}\right. \\
& \left.+\frac{x_{\bullet 2} \cdot x_{\diamond 2}+x_{\bullet 2} \cdot x_{\diamond 4}+x_{\bullet 2} \cdot x_{\diamond 10}+x_{\bullet 4} \cdot x_{\diamond 2}+x_{\bullet 4} \cdot x_{\diamond 4}+x_{\bullet 4} \cdot x_{\diamond 10}+x_{\bullet 10} \cdot x_{\diamond 2}+x_{\bullet 10} \cdot x_{\diamond 4}+x_{\bullet 10} \cdot x_{\diamond 10}}{4}\right) .
\end{aligned}
$$

$$
\begin{aligned}
\bar{W} x_{\bullet 3}^{\prime} & =\frac{W_{3}}{2}\left(x_{\bullet 3} \cdot x_{\diamond 3}+\frac{x_{\bullet 2} \cdot x_{\diamond 3}+x_{\bullet 3} \cdot x_{\diamond 2}+x_{\bullet 3} \cdot x_{\diamond 5}+x_{\bullet 3} \cdot x_{\diamond 6}+x_{\bullet 5} \cdot x_{\diamond 3}+x_{\bullet 6} \cdot x_{\diamond 3}}{2}\right. \\
& \left.+\frac{x_{\bullet 2} \cdot x_{\diamond 2}+x_{\bullet 2} \cdot x_{\diamond 5}+x_{\bullet 2} \cdot x_{\diamond 6}+x_{\bullet 5} \cdot x_{\diamond 2}+x_{\bullet 5} \cdot x_{\diamond 5}+x_{\bullet 5} \cdot x_{\diamond 6}+x_{\bullet 6} \cdot x_{\diamond 2}+x_{\bullet 6} \cdot x_{\diamond 5}+x_{\bullet 6} \cdot x_{\diamond 6}}{4}\right) .
\end{aligned}
$$

$$
\begin{aligned}
\bar{W} x_{\bullet 7}^{\prime} & =\frac{W_{7}}{2}\left(x_{\bullet 7} \cdot x_{\diamond 7}+\frac{x_{\bullet 4} \cdot x_{\diamond 7}+x_{\bullet 5} \cdot x_{\diamond 7}+x_{\bullet 7} \cdot x_{\diamond 5}+x_{\bullet 7} \cdot x_{\diamond 8}+x_{\bullet 8} \cdot x_{\diamond 7}}{2}\right. \\
& \left.+\frac{x_{\bullet 4} \cdot x_{\diamond 4}+x_{\bullet 4} \cdot x_{\diamond 5}+x_{\bullet 4} \cdot x_{\diamond 8}+x_{\bullet 5} \cdot x_{\diamond 4}+x_{\bullet 5} \cdot x_{\diamond 5}+x_{\bullet 5} \cdot x_{\diamond 8}+x_{\bullet 8} \cdot x_{\diamond 4}+x_{\bullet 8} \cdot x_{\diamond 5}+x_{\bullet 8} \cdot x_{\diamond 8}}{4}\right) .
\end{aligned}
$$

$$
\begin{aligned}
\bar{W} x_{\bullet 9}^{\prime} & =\frac{W_{9}}{2}\left(x_{\bullet 9} \cdot x_{\diamond 9}+\frac{x_{\bullet 6} \cdot x_{\diamond 9}+x_{\bullet 8} \cdot x_{\diamond 9}+x_{\bullet 9} \cdot x_{\diamond 6}+x_{\bullet 9} \cdot x_{\diamond 8}+x_{\bullet 9} \cdot x_{\diamond 10}+x_{\bullet 10} \cdot x_{\diamond 8}}{2}\right. \\
& \left.+\frac{x_{\bullet 6} \cdot x_{\diamond 6}+x_{\bullet 6} \cdot x_{\diamond 8}+x_{\bullet 10} \cdot x_{\diamond 8}+x_{\bullet 8} \cdot x_{\diamond 6}+x_{\bullet 8} \cdot x_{\diamond 8}+x_{\bullet 8} \cdot x_{\diamond 10}+x_{\bullet 10} \cdot x_{\diamond 6}+x_{\bullet 10} \cdot x_{\diamond 9}+x_{\bullet 10} \cdot x_{\diamond 10}}{4}\right) .
\end{aligned}
$$

Frequency of the single heterozygotes will be:

$$
\begin{aligned}
\bar{W} x_{\bullet 2}^{\prime} & =\frac{W_{2}}{2}\left(x_{\bullet 1} \cdot x_{\diamond 3}+x_{\bullet 3} \cdot x_{\diamond 1}+\frac{x_{\bullet 1} \cdot x_{\diamond 2}+x_{\bullet 1} \cdot x_{\diamond 5}+x_{\bullet 1} \cdot x_{\diamond 6}+x_{\bullet 2} \cdot x_{\diamond 1}+x_{\bullet 2} \cdot x_{\diamond 2}+x_{\bullet 2} \cdot x_{\diamond 3}+x_{\bullet 3} \cdot x_{\diamond 2}}{2}\right. \\
& +\frac{x_{\bullet 3} \cdot x_{\diamond 4}+x_{\bullet 3} \cdot x_{\diamond 10}+x_{\bullet 4} \cdot x_{\diamond 3}+x_{\bullet 5} \cdot x_{\diamond 1}+x_{\bullet 5} \cdot x_{\diamond 2}+x_{\bullet 6} \cdot x_{\diamond 1}+x_{\bullet 10} \cdot x_{\diamond 2}}{2} \\
& +\frac{x_{\bullet 2} \cdot x_{\diamond 4}+x_{\bullet 2} \cdot x_{\diamond 5}+x_{\bullet 2} \cdot x_{\diamond 6}+x_{\bullet 2} \cdot x_{\diamond 10}+x_{\bullet 4} \cdot x_{\diamond 2}+x_{\bullet 4} \cdot x_{\diamond 5}+x_{\bullet 4} \cdot x_{\diamond 6}+x_{\bullet 5} \cdot x_{\diamond 4}+x_{\bullet 5} \cdot x_{\diamond 10}}{4} \\
& \left.+\frac{x_{\bullet 6} \cdot x_{\diamond 2}+x_{\bullet 6} \cdot x_{\diamond 4}+x_{\bullet 6} \cdot x_{\diamond 10}+x_{\bullet 10} \cdot x_{\diamond 2}+x_{\bullet 10} \cdot x_{\diamond 5}+x_{\bullet 10} \cdot x_{\diamond 6}}{4}\right) .
\end{aligned}
$$


bioRxiv preprint doi: https://doi.org/10.1101/2020.01.03.892810; this version posted January 3, 2020. The copyright holder for this preprint (which was not certified by peer review) is the author/funder, who has granted bioRxiv a license to display the preprint in perpetuity. It is made available under aCC-BY-NC-ND 4.0 International license.

$$
\begin{aligned}
\bar{W} x_{\bullet 4}^{\prime} & =\frac{W_{4}}{2}\left(x_{\bullet 1} \cdot x_{\diamond 7}+x_{\bullet 7} \cdot x_{\diamond 1}+\frac{x_{\bullet 1} \cdot x_{\diamond 4}+x_{\bullet 1} \cdot x_{\diamond 5}+x_{\bullet 1} \cdot x_{\diamond 8}+x_{\bullet 4} \cdot x_{\diamond 1}+x_{\bullet 4} \cdot x_{\diamond 4}+x_{\bullet 4} \cdot x_{\diamond 7}+x_{\bullet 5} \cdot x_{\diamond 1}}{2}\right. \\
& +\frac{x_{\bullet 7} \cdot x_{\diamond 2}+x_{\bullet 7} \cdot x_{\diamond 4}+x_{\bullet 7} \cdot x_{\diamond 10}+x_{\bullet 8} \cdot x_{\diamond 1}+x_{\bullet 10} \cdot x_{\diamond 7}+x_{\bullet 10} \cdot x_{\diamond 8}}{2} \\
& +\frac{x_{\bullet 2} \cdot x_{\diamond 4}+x_{\bullet 2} \cdot x_{\diamond 5}+x_{\bullet 2} \cdot x_{\diamond 7}+x_{\bullet 2} \cdot x_{\diamond 8}+x_{\bullet 4} \cdot x_{\diamond 2}+x_{\bullet 4} \cdot x_{\diamond 5}+x_{\bullet 4} \cdot x_{\diamond 8}+x_{\bullet 4} \cdot x_{\diamond 10}+x_{\bullet 5} \cdot x_{\diamond 2}+x_{\bullet 5} \cdot x_{\diamond 4}}{4} \\
& \left.+\frac{x_{\bullet 5} \cdot x_{\diamond 10}+x_{\bullet 8} \cdot x_{\diamond 2}+x_{\bullet 8} \cdot x_{\diamond 4}+x_{\bullet 8} \cdot x_{\diamond 10}+x_{\bullet 10} \cdot x_{\diamond 4}+x_{\bullet 10} \cdot x_{\diamond 5}}{4}\right) .
\end{aligned}
$$

$$
\begin{aligned}
& \bar{W} x_{\bullet 6}^{\prime}=\frac{W_{6}}{2}\left(x_{\bullet 9} \cdot x_{\diamond 3}+x_{\bullet 3} . x_{\diamond 9}+\frac{x_{\bullet 2} . x_{\diamond 9}+x_{\bullet 3} . x_{\diamond 6}+x_{\bullet 3} \cdot x_{\diamond 8}+x_{\bullet 3} \cdot x_{\diamond 10}+x_{\bullet 5} \cdot x_{\diamond 9}+x_{\bullet 6} \cdot x_{\diamond 3}+x_{\bullet 6} \cdot x_{\diamond 6}}{2}\right. \\
& +\frac{x_{\bullet 6} \cdot x_{\diamond 9}+x_{\bullet 8} \cdot x_{\diamond 3}+x_{\bullet 9} \cdot x_{\diamond 2}+x_{\bullet 9} \cdot x_{\diamond 5}+x_{\bullet 9} \cdot x_{\diamond 6}+x_{\bullet 10} \cdot x_{\diamond 3}}{2} \\
& +\frac{x_{\bullet 2} . x_{\diamond 6}+x_{\bullet 2} . x_{\diamond 8}+x_{\bullet 2} . x_{\diamond 10}+x_{\bullet 5} \cdot x_{\diamond 6}+x_{\bullet 5} \cdot x_{\diamond 8}+x_{\bullet 5} \cdot x_{\diamond 10}+x_{\bullet 6} \cdot x_{\diamond 2}+x_{\bullet 6} \cdot x_{\diamond 5}+x_{\bullet 6} \cdot x_{\diamond 8}}{4} \\
& \left.+\frac{x_{\bullet 6} \cdot x_{\diamond 10}+x_{\bullet 8} \cdot x_{\diamond 2}+x_{\bullet 8} \cdot x_{\diamond 5}+x_{\bullet 8} \cdot x_{\diamond 6}+x_{\bullet 10} \cdot x_{\diamond 2}+x_{\bullet 10} \cdot x_{\diamond 5}+x_{\bullet 10} \cdot x_{\diamond 6}}{4}\right) .
\end{aligned}
$$

$$
\begin{aligned}
\bar{W} x_{\bullet 8}^{\prime} & =\frac{W_{8}}{2}\left(x_{\bullet 7} \cdot x_{\diamond 9}+x_{\bullet 9} \cdot x_{\diamond 7}+\frac{x_{\bullet 4} \cdot x_{\diamond 9}+x_{\bullet 5} \cdot x_{\diamond 9}+x_{\bullet 6} \cdot x_{\diamond 7}+x_{\bullet 7} \cdot x_{\diamond 6}+x_{\bullet 7} \cdot x_{\diamond 8}+x_{\bullet 7} \cdot x_{\diamond 10}+x_{\bullet 8} \cdot x_{\diamond 7}}{2}\right. \\
& +\frac{x_{\bullet 8} \cdot x_{\diamond 8}+x_{\bullet 8} \cdot x_{\diamond 9}+x_{\bullet 8} \cdot x_{\diamond 10}+x_{\bullet 9} \cdot x_{\diamond 4}+x_{\bullet 9} \cdot x_{\diamond 5}+x_{\bullet 9} \cdot x_{\diamond 8}+x_{\bullet 10} \cdot x_{\diamond 7}}{2} \\
& +\frac{x_{\bullet 4} \cdot x_{\diamond 6}+x_{\bullet 4} \cdot x_{\diamond 8}+x_{\bullet 4} \cdot x_{\diamond 10}+x_{\bullet 5} \cdot x_{\diamond 6}+x_{\bullet 5} \cdot x_{\diamond 8}+x_{\bullet 5} \cdot x_{\diamond 10}+x_{\bullet 6} \cdot x_{\diamond 4}+x_{\bullet 6} \cdot x_{\diamond 5}+x_{\bullet 6} \cdot x_{\diamond 8}}{4} \\
& \left.+\frac{x_{\bullet 8} \cdot x_{\diamond 4}+x_{\bullet 8} \cdot x_{\diamond 5}+x_{\bullet 8} \cdot x_{\diamond 6}+x_{\bullet 10} \cdot x_{\diamond 4}+x_{\bullet 10} \cdot x_{\diamond 5}+x_{\bullet 10} \cdot x_{\diamond 8}}{4}\right) .
\end{aligned}
$$

Frequency of the double heterozygotes will be:

$$
\begin{aligned}
\bar{W} x_{\bullet 5}^{\prime} & =\frac{W_{5}}{2}\left(x_{\bullet 3} \cdot x_{\diamond 7}+x_{\bullet 7} \cdot x_{\diamond 3}+\frac{x_{\bullet 2} \cdot x_{\diamond 7}+x_{\bullet 3} \cdot x_{\diamond 4}+x_{\bullet 3} \cdot x_{\diamond 5}+x_{\bullet 3} \cdot x_{\diamond 8}+x_{\bullet 4} \cdot x_{\diamond 3}+x_{\bullet 5} \cdot x_{\diamond 3}}{2}\right. \\
& +\frac{x_{\bullet 5} \cdot x_{\diamond 5}+x_{\bullet 5} \cdot x_{\diamond 7}+x_{\bullet 6} \cdot x_{\diamond 5}+x_{\bullet 7} \cdot x_{\diamond 2}+x_{\bullet 7} \cdot x_{\diamond 5}+x_{\bullet 7} \cdot x_{\diamond 6}+x_{\bullet 8} \cdot x_{\diamond 3}}{2} \\
& +\frac{x_{\bullet 2} \cdot x_{\diamond 4}+x_{\bullet 2} \cdot x_{\diamond 5}+x_{\bullet 2} \cdot x_{\diamond 8}+x_{\bullet 4} \cdot x_{\diamond 2}+x_{\bullet 4} \cdot x_{\diamond 5}+x_{\bullet 4} \cdot x_{\diamond 6}+x_{\bullet 5} \cdot x_{\diamond 2}+x_{\bullet 5} \cdot x_{\diamond 4}+x_{\bullet 5} \cdot x_{\diamond 6}}{4} \\
& \left.+\frac{x_{\bullet 5} \cdot x_{\diamond 8}+x_{\bullet 6} \cdot x_{\diamond 4}+x_{\bullet 6} \cdot x_{\diamond 7}+x_{\bullet 6} \cdot x_{\diamond 8}+x_{\bullet 8} \cdot x_{\diamond 2}+x_{\bullet 8} \cdot x_{\diamond 5}+x_{\bullet 8} \cdot x_{\diamond 6}}{4}\right) .
\end{aligned}
$$




$$
\begin{aligned}
\bar{W} x_{\bullet 10}^{\prime} & =\frac{W_{10}}{2}\left(x_{\bullet 1} \cdot x_{\diamond 9}+x_{\bullet 9} \cdot x_{\diamond 1}+\frac{x_{\bullet 1} \cdot x_{\diamond 6}+x_{\bullet 1} \cdot x_{\diamond 8}+x_{\bullet 1} \cdot x_{\diamond 10}+x_{\bullet 2} \cdot x_{\diamond 8}+x_{\bullet 4} \cdot x_{\diamond 8}+x_{\bullet 6} \cdot x_{\diamond 1}+x_{\bullet 8} \cdot x_{\diamond 1}}{2}\right. \\
& +\frac{x_{\bullet 9} \cdot x_{\diamond 2}+x_{\bullet 9} \cdot x_{\diamond 4}+x_{\bullet 9} \cdot x_{\diamond 10}+x_{\bullet 10} \cdot x_{\diamond 1}+x_{\bullet 10} \cdot x_{\diamond 9}+x_{\bullet 10} \cdot x_{\diamond 10}}{2} \\
& +\frac{x_{\bullet 2} \cdot x_{\diamond 6}+x_{\bullet 2} \cdot x_{\diamond 9}+x_{\bullet 2} \cdot x_{\diamond 10}+x_{\bullet 4} \cdot x_{\diamond 6}+x_{\bullet 4} \cdot x_{\diamond 9}+x_{\bullet 4} \cdot x_{\diamond 10}+x_{\bullet 6} \cdot x_{\diamond 2}+x_{\bullet 6} \cdot x_{\diamond 4}}{4} \\
& \left.+\frac{x_{\bullet 6} \cdot x_{\diamond 10}+x_{\bullet 8} \cdot x_{\diamond 2}+x_{\bullet 8} \cdot x_{\diamond 4}+x_{\bullet 8} \cdot x_{\diamond 10}+x_{\bullet 10} \cdot x_{\diamond 2}+x_{\bullet 10} \cdot x_{\diamond 4}+x_{\bullet 10} \cdot x_{\diamond 6}+x_{\bullet 10} \cdot x_{\diamond 8}}{4}\right) .
\end{aligned}
$$

380

Here, the $W_{i}$ s are the fitnesses of each genotype $i$ with frequency $x_{i}$ and $\bar{W}$ is their mean fitness.

381 Similarly, we can obtain the frequencies of the genotypes in Sex 2. 


\section{Ratio of Heterozygotes/ Homozygotes in Sex 1}

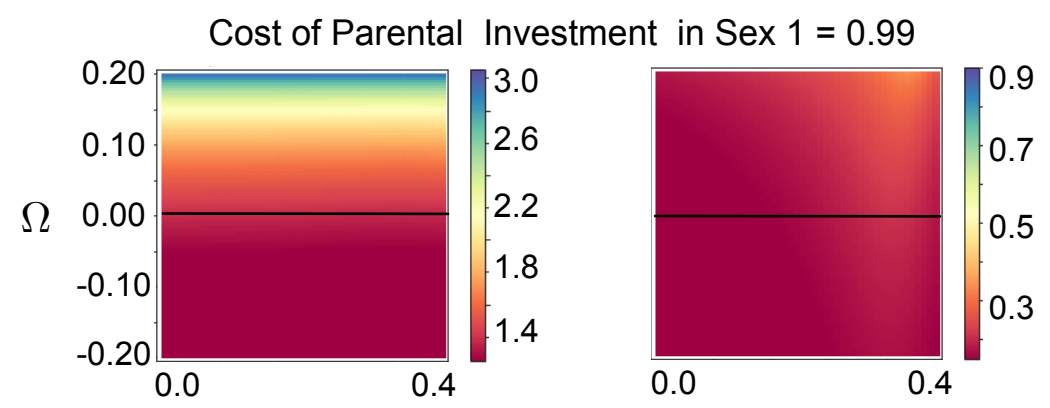

Cost of Parental Investment in Sex $1=0.66$
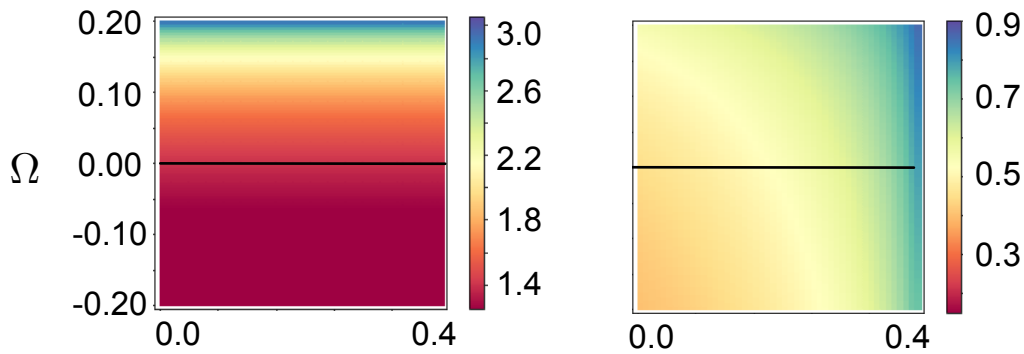

Cost of Parental Investment in Sex $1=0.33$
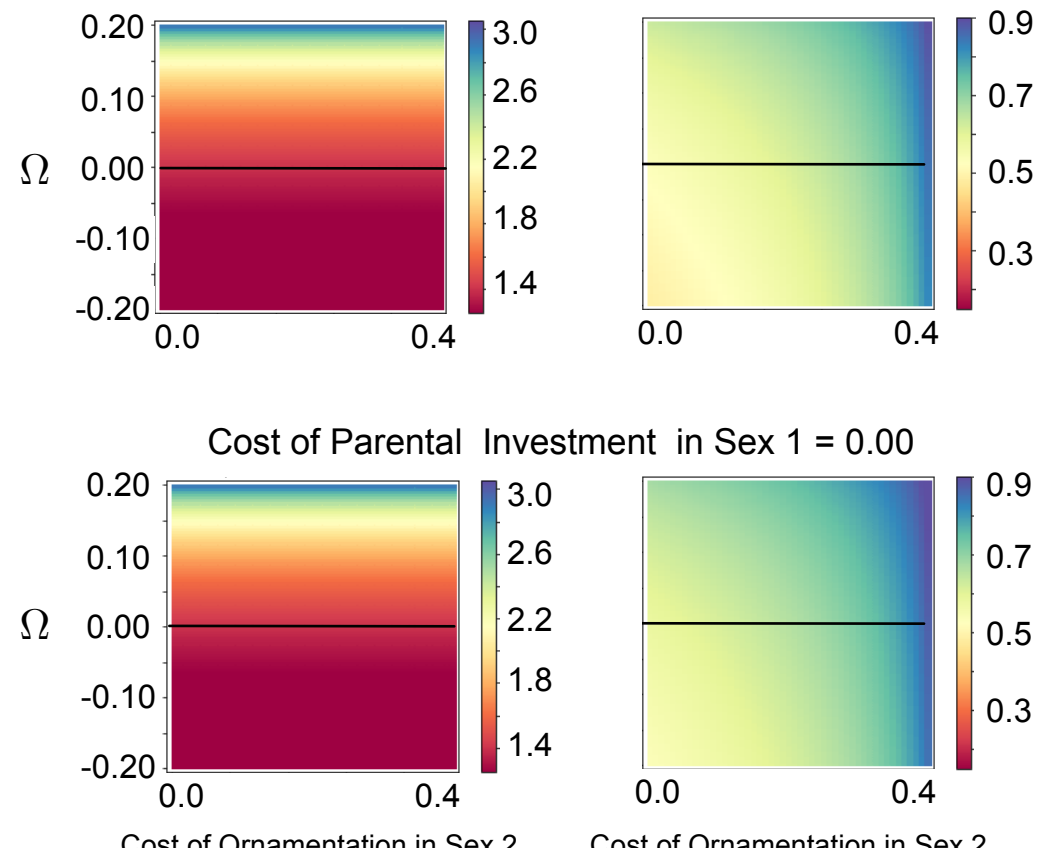

Figure S.3: Ratio of Heterozygotes: Homozygotes in Sex 1 and the frequency of Sex 2 for a full range of $\Omega$. The parameter $\Omega$ is a measure of the sex difference in immune response through sexual conflict within the MHC as shown in Figure 2. in the main article. It represents the sex-specific fitness effect of Sex 1 relative to Sex 2 . When $\Omega=0$, there is so sexspecific difference in immune response. There is no effect of ornamentation and parental investment (PI) on the ratio of allele diversity. However, $\Omega$ has an effect on this ratio. All factors: coat of PI, cost of ornamentation and $\Omega$ have an effect on the frequency of the sexes. Thought the effect of $\Omega$ is not profound, the cost of ornamentation in Sex 2 and cost of PI in Sex 1 reduce their frequency, respectively. 


\section{Ratio of Heterozygotes/ Homozygotes in Sex 1}

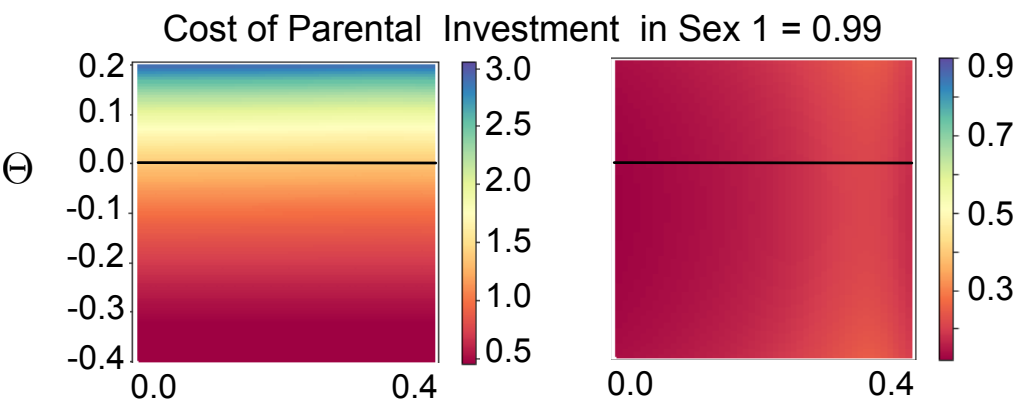

Cost of Parental Investment in Sex $1=0.66$
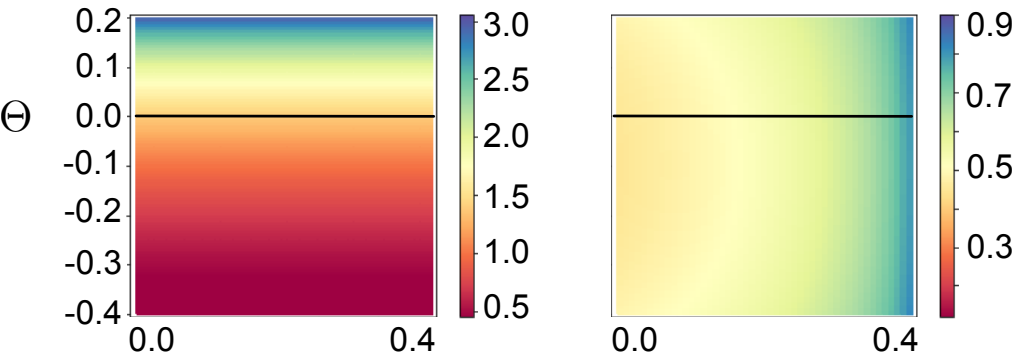

Cost of Parental Investment in Sex $1=0.33$
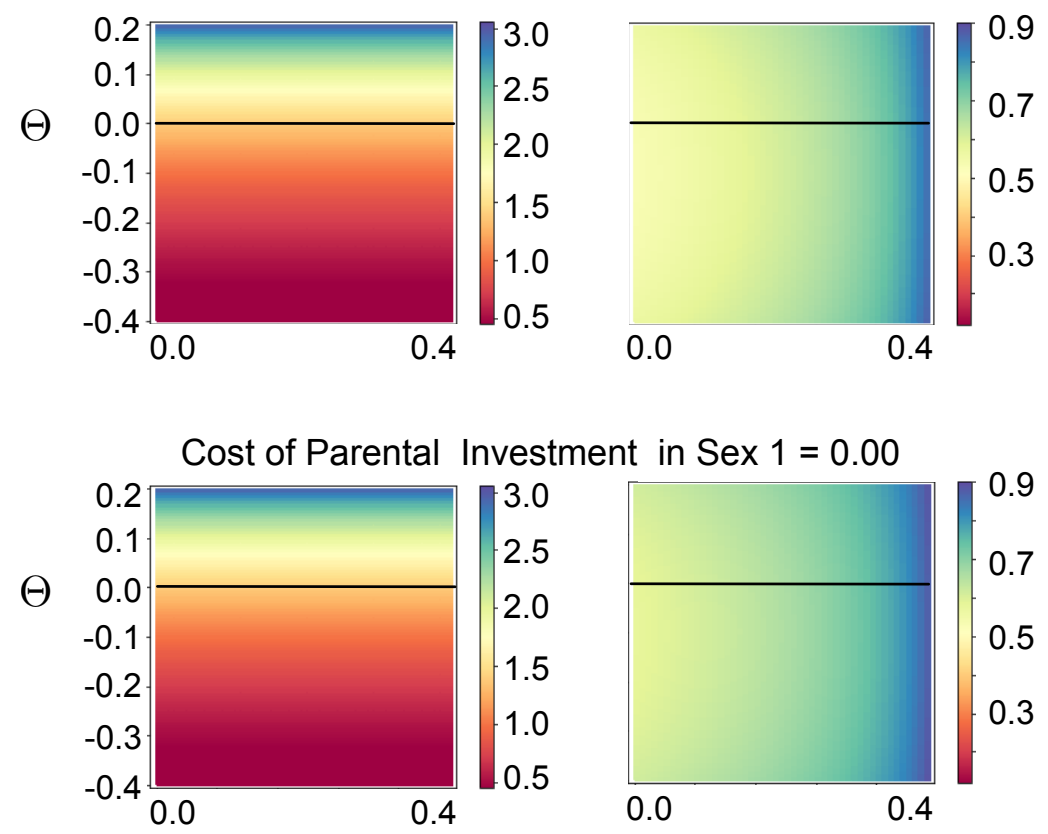

Cost of Ornamentation in Sex 2

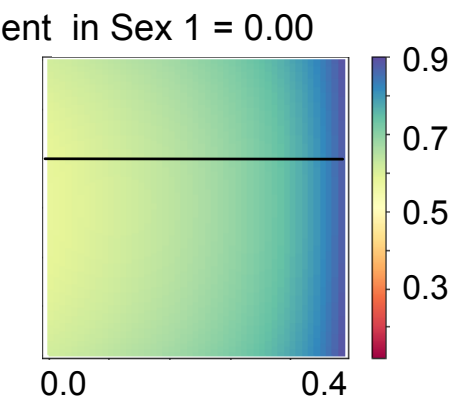

Cost of Ornamentation in Sex 2

Figure S.4: Ratio of Heterozygotes: Homozygotes in Sex 1 and the frequency of Sex 2 for a full range of $\Theta$. The parameter $\Theta$ is a measure of the sex difference in immune response through sexual conflict within the MHC as shown in Figure 2. in the main article. It represents the effect of allelic diversity on sex-specific fitness of Sex 1 relative to Sex 2 . When $\Theta=0$, there is so sex-specific difference in immune response. The parameter $\Theta$ has an effect on the allele diversity ratio. But there is no effect of ornamentation and parental investment $(\mathrm{PI})$ on this ratio. There is no effect of $\Theta$ on the frequency of Sex 1. The cost of ornamentation in Sex 2 increases the frequency of Sex 1 while the cost of PI decreases its frequency. 
Ratio of Heterozygotes/ Homozygotes in Sex 1

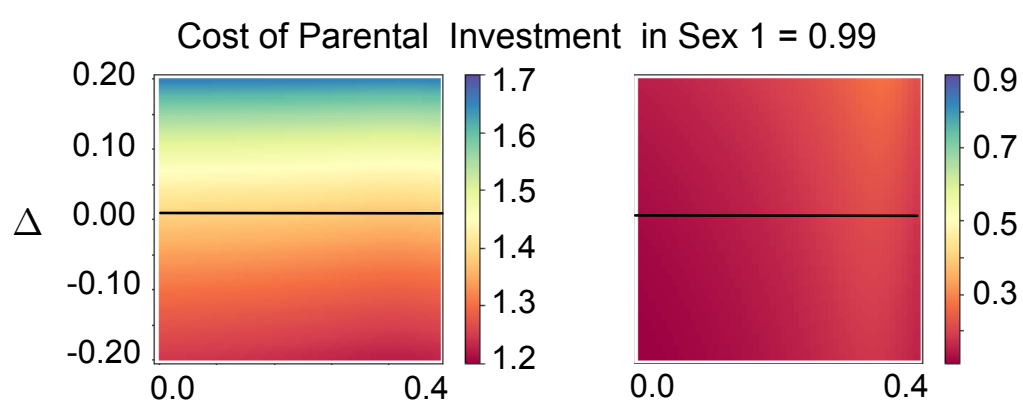

Cost of Parental Investment in Sex $1=0.66$
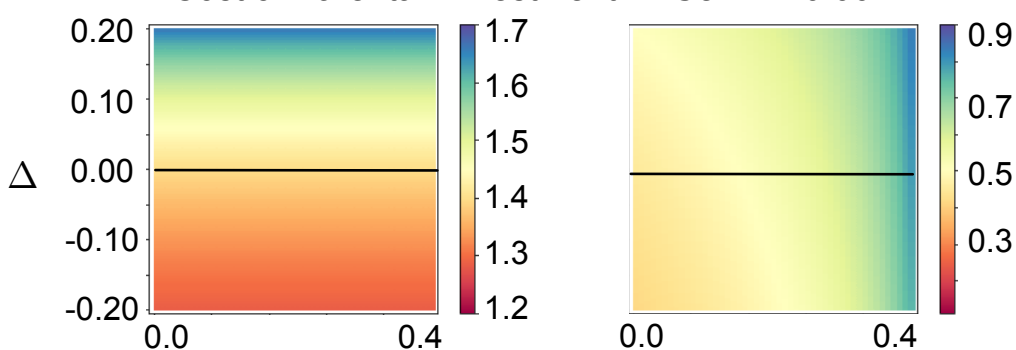

Cost of Parental Investment in Sex $1=0.33$
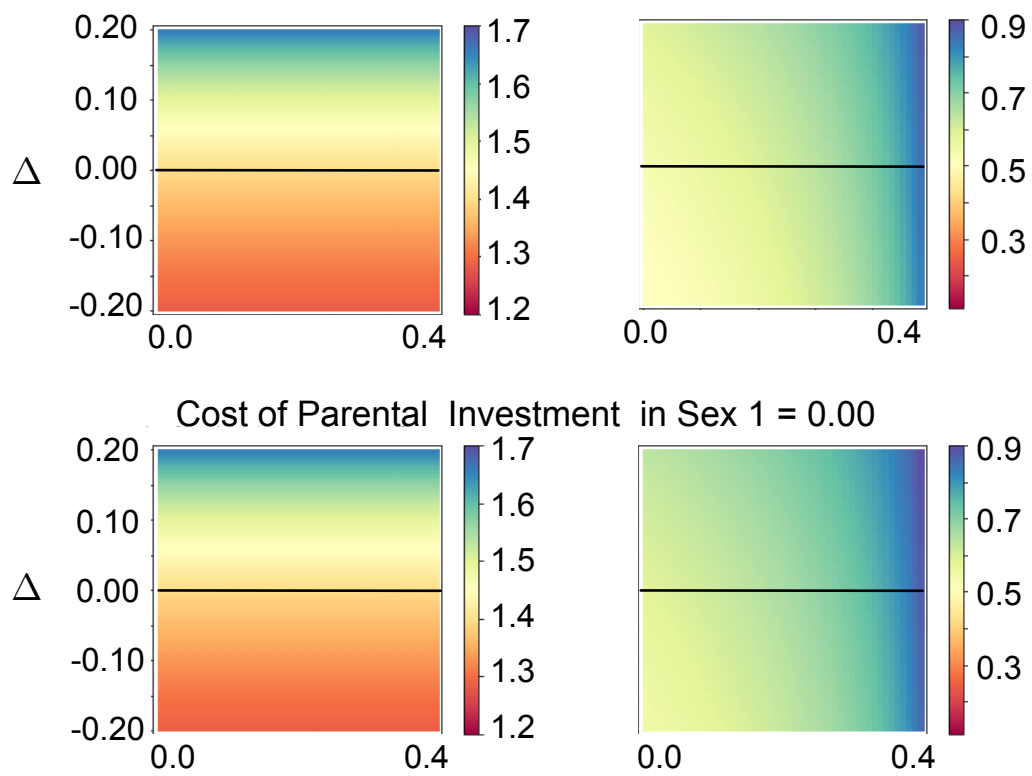

Cost of Ornamentation in Sex 2

Figure S.5: Ratio of Heterozygotes: Homozygotes in Sex 1 and the frequency of Sex 2 for a full range of $\Delta$. The parameter $\Delta$ is a measure of the sex difference in immune response through sexual conflict within the MHC as shown in Figure 2. in the main article. It represents the sex-specific fitness effect (that also includes the effect of diversity on sex-specific fitness) of Sex 1 relative to Sex 2 . When $\Delta=0$, there is so sex-specific difference in immune response. There is no effect of ornamentation and parental investment $(\mathrm{PI})$ on the ratio of allele diversity. But $\Delta$ has an effect on this ratio. As observed in the previous figure, here too, the cost of ornamentation in Sex 2 and cost of PI in Sex 1 reduce their frequency, respectively while the effect of $\Delta$ is not as profound. 


\section{References}

Alonzo, S. H., 2002. State-dependent habitat selection games between predators and prey: the importance of behavioural interactions and expected lifetime reproductive success. Evolutionary Ecology Research 4 (5), 759-778.

Alonzo, S. H., 2010. Social and coevolutionary feedbacks between mating and parental investment. Trends in Ecology \& Evolution 25 (2), 99-108.

Andersson, M., Simmons, L. W., 2006. Sexual selection and mate choice. Trends in ecology \& evolution 21 (6), 296-302.

Apanius, V., Penn, D., Slev, P., Ruff, L., Potts., W., 1997. The nature of selection on the major histocompatibility complex. Critical Reviews in Immunology 17, 179-224.

Austad, S. N., 2006. Why women live longer than men: sex differences in longevity. Gender medicine 3 (2), 79-92.

Bateman, A., 1948. Intra-sexual selection in drosophila. Heredity 2 (3), 349-68.

Bell, G., 1978. The evolution of anisogamy. Journal of Theoretical Biology 73 (2), 247-270.

Berglund, A., Rosenqvist, G., 2003. Sex role reversal in pipefish. Advances in the Study of Behavior 32, 131-168.

Berglund, A., Rosenqvist, G., Bernet, P., 1997. Ornamentation predicts reproductive success in female pipefish. Behavioral Ecology and Sociobiology 40 (3), 145-150.

Berglund, A., Rosenqvist, G., Svensson, I., 1986. Reversed sex roles and parental energy investment in zygotes of two pipefish (syngnathidae) species. Marine Ecology Progress Series, 209-215.

Bolnick, D. I., Doebeli, M., 2003. Sexual dimorphism and adaptive speciation: two sides of the same ecological coin. Evolution 57 (11), 2433-2449.

Carranza, J., Álvarez, F., Redondo, T., 1990. Territoriality as a mating strategy in red deer. Animal Behaviour 40 (1), 79-88.

Chandra, R. K., 1983. Nutrition, Immunity, and Infection - Present Knowledge and Future-Directions. Lancet 1 (8326), 688-691.

Chapman, T., Partridge, L., 1996. Female fitness in drosophila melanogaster: an interaction between the effect of nutrition and of encounter rate with males. Proceedings of the Royal Society of London. Series B: Biological Sciences 263 (1371), 755-759.

Chen, W., Gracia-Lázaro, C., Li, Z., Wang, L., Moreno, Y., 2017. Evolutionary dynamics of n-person hawk-dove games. Scientific reports 7 (1), 4800.

Cockburn, A., 2006. Prevalence of different modes of parental care in birds. Proceedings of the Royal Society B: Biological Sciences 273 (1592), 1375-1383.

Crow, J. F., Kimura, M., 1970. An Introduction to Population Genetics Theory. Harper and Row, New York.

Daly, M., 1978. The cost of mating. The American Naturalist 112 (986), 771-774. 
De Lisle, S. P., 2019. Understanding the evolution of ecological sex differences: Integrating character displacement and the darwin-bateman paradigm. Evolution Letters 0 (0).

Doebeli, M., Ispolatov, Y., Simon, B., 2017. Towards a mechanistic foundation of evolutionary theory. eLife 6, e23804.

Eizaguirre, C., Yeates, S. E., Lenz, T. L., Kalbe, M., Milinski, M., 2009. Mhc-based mate choice combines good genes and maintenance of mhc polymorphism. Molecular Ecology 18 (15), 33163329.

Fleming, I. A., Hindar, K., MjÖlnerÖd, I. B., Jonsson, B., Balstad, T., Lamberg, A., 2000. Lifetime success and interactions of farm salmon invading a native population. Proceedings of the Royal Society of London. Series B: Biological Sciences 267 (1452), 1517-1523.

Forbes, M. R., 2007. On sex differences in optimal immunity. Trends in ecology \& evolution 22 (3), 111-113.

Freeman, S., Herron, J. C., 2007. Evolutionary analysis. No. QH 366.2. F73 2007. Pearson Prentice Hall Upper Saddle River, NJ.

Gokhale, C. S., Reeves, R. G., Reed, F. A., 2014. Dynamics of a combined medea-underdominant population transformation system. BMC Evolutionary Biology 14 (1), 98.

Gokhale, C. S., Traulsen, A., 2014. Evolutionary multiplayer games. Dynamic Games and Applications 4, 468-488.

Gross, M. R., Sargent, R. C., 1985. The evolution of male and female parental care in fishes. American Zoologist 25 (3), 807-822.

Hagen, D., Gilbertson, L., 1973. Selective predation and the intensity of selection acting upon the lateral plates of threespine sticklebacks. Heredity 30 (3), 273.

Hayward, A., Gillooly, J. F., 2011. The cost of sex: quantifying energetic investment in gamete production by males and females. PLoS One 6 (1), e16557.

Hedrick, A. V., Temeles, E. J., 1989. The evolution of sexual dimorphism in animals: hypotheses and tests. Trends in Ecology \& Evolution 4 (5), 136-138.

Henshaw, J. M., Fromhage, L., Jones, A. G., 2019. Sex roles and the evolution of parental care specialization. Proceedings of the Royal Society B 286 (1909), 20191312.

Hillgarth, N., Wingfield, J. C., 1997. Testosterone and immunosuppression in vertebrates: implications for parasite-mediated sexual selection. In: Parasites and pathogens. Springer, pp. 143-155.

Hofbauer, J., Sigmund, K., 1998. Evolutionary Games and Population Dynamics. Cambridge University Press, Cambridge, UK.

Kalbe, M., Eizaguirre, C., Dankert, I., Reusch, T. B. H., Sommerfeld, R. D., Wegner, K. M., Milinski, M., 2009. Lifetime reproductive success is maximized with optimal major histocompatibility complex diversity. Proceedings of the Royal Society B 276, 925-934.

Kelly, N., Alonzo, S., 2010. Does a trade-off between current reproductive success and survival affect the honesty of male signalling in species with male parental care? Journal of evolutionary biology 23 (11), 2461-2473. 
Kirkpatrick, M., 1982. Sexual selection and the evolution of female choice. Evolution 36 (1), 1-12.

Kokko, H., Jennions, M., 2003. It takes two to tango. Trends in Ecology \& Evolution 18 (3) 103-104.

Kokko, H., Jennions, M. D., 2008. Parental investment, sexual selection and sex ratios. Journal of evolutionary biology 21 (4), 919-948.

Kurtz, J., 2007. The correlation between immunocompetence and an ornament trait changes over lifetime in panorpa vulgaris scorpionflies. Zoology 110 (5), 336-343.

Liker, A., Freckleton, R. P., Remeš, V., Székely, T., 2015. Sex differences in parental care: Gametic investment, sexual selection, and social environment. Evolution 69 (11), 2862-2875.

Liker, A., Freckleton, R. P., Székely, T., 2013. The evolution of sex roles in birds is related to adult sex ratio. Nature Communications 4, 1587.

Lin, T., Zhang, D., Liu, X., Xiao, D., 2016. Parental care improves immunity in the seahorse (hippocampus erectus). Fish \& Shellfish Immunology 58, 554-562.

Love, O. P., Salvante, K. G., Dale, J., Williams, T. D., 2008. Sex-specific variability in the immune system across life-history stages. The American Naturalist 172 (3), E99-E112.

Martin, T. E., 1992. Interaction of nest predation and food limitation in reproductive strategies. In: Current ornithology. Springer, pp. 163-197.

May, R. C., 2007. Gender, immunity and the regulation of longevity. Bioessays 29 (8), 795-802.

Maynard Smith, J., 1986. Evolutionary game theory. Physica D: Nonlinear Phenomena 22 (1), 43-49.

Milinski, M., 2006. The major histocompatibility complex, sexual selection, and mate choice. Annual Review of Ecology, Evolution, and Systematics 37, 159-186.

Mobley, K. B., Granroth-Wilding, H., Ellmen, M., Vähä, J.-P., Aykanat, T., Johnston, S. E., Orell, P., Erkinaro, J., Primmer, C. R., 2019. Home ground advantage: Local atlantic salmon have higher reproductive fitness than dispersers in the wild. Science advances 5 (2), eaav1112.

Moore, A. J., 1990. The evolution of sexual dimorphism by sexual selection: the separate effects of intrasexual selection and intersexual selection. Evolution 44 (2), 315-331.

Nowak, M. A., Tarczy-Hornoch, K., Austyn, J. M., 1992. The optimal number of major histocompatibility complex molecules in an individual. Proceedings of the National Academy of Sciences USA 89 (22), 10896-10899.

Nunn, C. L., Lindenfors, P., Pursall, E. R., Rolff, J., 2008. On sexual dimorphism in immune function. Philosophical Transactions of the Royal Society B: Biological Sciences 364 (1513), 61-69.

Oertelt-Prigione, S., 2012. The influence of sex and gender on the immune response. Autoimmunity reviews 11 (6-7), A479-A485.

Otto, S. P., Day, T., 2007. A Biologist's Guide to Mathematical Modeling in Ecology and Evolution. Princeton Univ. Press, Princeton, NJ.

Perrone Jr, M., Zaret, T. M., 1979. Parental care patterns of fishes. The American Naturalist 113 (3), 351-361. 
Petrie, M., Tim, H., Carolyn, S., 1991. Peahens prefer peacocks with elaborate trains. Animal Behaviour 41 (2), 323-331.

Pipoly, I., Bókony, V., Kirkpatrick, M., Donald, P. F., Székely, T., Liker, A., 2015. The genetic sex-determination system predicts adult sex ratios in tetrapods. Nature 527 (7576), 91.

Pusey, A., Williams, J., Goodall, J., 1997. The influence of dominance rank on the reproductive success of female chimpanzees. Science 277 (5327), 828-831.

Roth, O., Scharsack, J., Keller, I., Reusch, T. B., 2011. Bateman's principle and immunity in a sex-role reversed pipefish. Journal of evolutionary biology 24 (7), 1410-1420.

Roved, J., 2019. MHC polymorphism in a songbird.

Roved, J., Hansson, B., Tarka, M., Hasselquist, D., Westerdahl, H., 2018. Evidence for sexual conflict over major histocompatibility complex diversity in a wild songbird. Proceedings of the Royal Society B: Biological Sciences 285 (1884), 20180841.

Roved, J., Westerdahl, H., Hasselquist, D., 2017. Sex differences in immune responses: hormonal effects, antagonistic selection, and evolutionary consequences. Hormones and Behavior 88, 95105.

Schuster, P., Sigmund, K., 1983. Replicator dynamics. Journal of Theoretical Biology 100, 533-538.

Sigmund, K., Nowak, M. A., 1999. Evolutionary game theory. Current Biology 9 (14), R503-R505.

Smith, C., Wootton, R., 1999. Parental energy expenditure of the male three-spined stickleback. Journal of Fish Biology 54 (5), 1132-1136.

Stoehr, A. M., Kokko, H., 2006. Sexual dimorphism in immunocompetence: what does life-history theory predict? Behavioral Ecology 17 (5), 751-756.

Székely, T., Weissing, F. J., Komdeur, J., 2014. Adult sex ratio variation: implications for breeding system evolution. Journal of evolutionary biology 27 (8), 1500-1512.

Törnwall, J., Carey, A., Fox, R., Fox, H. S., 1999. Estrogen in autoimmunity: expression of estrogen receptors in thymic and autoimmune $t$ cells. The journal of gender-specific medicine: JGSM: the official journal of the Partnership for Women's Health at Columbia 2 (5), 33-40.

Trivers, R., 1972. Parental investment and sexual selection. Sexual Selection \& the Descent of Man, Aldine de Gruyter, New York, 136-179.

Trivers, R., 2002. Natural selection and social theory: Selected papers of Robert Trivers. Oxford University Press, USA.

Uekert, S. J., Akan, G., Evans, M. D., Li, Z., Roberg, K., Tisler, C., DaSilva, D., Anderson, E., Gangnon, R., Allen, D. B., et al., 2006. Sex-related differences in immune development and the expression of atopy in early childhood. Journal of allergy and clinical immunology 118 (6), $1375-1381$.

Vasconcelos, P., Rueffler, C., in press. How does joint evolution of consumer traits affect resource specialization? The American Naturalist. 
Venkateswaran, V. R., Gokhale, C. S., 2019. Evolutionary dynamics of complex multiple games. Proceedings of the Royal Society B: Biological Sciences 286 (1905), 20190900.

Vincent, A. C., Sadler, L. M., 1995. Faithful pair bonds in wild seahorses, hippocampus whitei. Animal behaviour 50 (6), 1557-1569.

Wade, M. J., Shuster, S. M., 2002. The evolution of parental care in the context of sexual selection: a critical reassessment of parental investment theory. The American Naturalist 160 (3), 285-292.

Whitacre, C. C., 2001. Sex differences in autoimmune disease. Nature immunology 2 (9), 777.

Woelfing, B., Traulsen, A., Milinski, M., Boehm, T., 2009. Does intra-individual major histocompatibility complex diversity keep a golden mean? Philosophical Transactions of the Royal Society B 364, 117-128.

Wong, B. B., Candolin, U., 2005. How is female mate choice affected by male competition? Biological Reviews 80 (4), 559-571.

Zahavi, A., 1977. The cost of honesty (further remarks on the handicap principle). Journal of theoretical Biology 67 (3), 603-605. 